\title{
A dual formulation of wavefield reconstruction inversion for large-scale seismic inversion
}

\author{
Gabrio Rizzuti $^{1} \quad$ Mathias Louboutin ${ }^{1} \quad$ Rongrong Wang $^{2}$ \\ Felix J. Herrmann ${ }^{1}$ \\ ${ }^{1}$ Georgia Institute of Technology, \\ ${ }^{2}$ Michigan State University
}

\section{Abstract}

Many of the seismic inversion techniques currently proposed that focus on robustness with respect to the background model choice are not apt to largescale 3D applications, and the methods that are computationally feasible for industrial problems, such as full waveform inversion, are notoriously bogged down by local minima and require adequate starting models. We propose a novel solution that is both scalable and less sensitive to starting model or modeling inaccuracies (e.g. stemming from inaccurate physical parameters that are kept fixed during inversion) when compared to full waveform inversion. It is based on a dual reformulation of the classical wavefield reconstruction inversion, whose empirical robustness with respect to local minima is well documented in the literature. Alas, the classical version is not suited to 3D, as it leverages expensive frequency-domain solvers for the wave equation. Our proposal, instead, allows the deployment of state-of-the-art time-domain finite-difference methods, and is computationally mature for industrial scale problems.

\section{Introduction}

Field-data applications of wave-equation based seismic inversion are limited by the computational complexity of large-scale 3D simulations, and by the tendency of traditional inversion techniques (such as full waveform inversion, FWI, Tarantola and Valette, 1982) to converge to unrealistic models. While considerable advances have been made to speed up wave equation solvers and avoid local minima, most of the currently proposed inversion methods do not take advantage of both these developments. While FWI is applied routinely in industry, techniques that are robust to local minima are typically limited to small problems. In this paper, we introduce a novel method that is fit for 3D large-scale deployment and relatively robust against spurious minima.

We adopt model extension methods that aim at counteracting local minimum stagnation, which are based on a reformulation of the original inverse problem 
into a higher dimensional space of unknowns (Symes, 2008). While beneficial for avoiding local minima, the inherent added complexity of these methods typically curtails large-scale applications. One notable example, and the basis for this work, is wavefield reconstruction inversion (WRI, van Leeuwen and Herrmann, 2013), which has demonstrated robustness towards local minima but is not well suited for 3D problems. Indeed, WRI involves the solution of an extended wave equation that is conventionally solved in the frequency domain with direct or iterative methods, although not efficiently for large grid sizes. Many other methods related to WRI share similar limitations (Huang et al., 2017, 2018; Aghamiry et al., 2019).

We propose a principled Lagrangian reformulation of classical WRI, referred to as WRI* throughout the text, which naturally lends itself to time-domain solvers, hence large-scale applications. The reformulation is based on minimizing the wave equation error and interpreting the data misfit as a constraint. The resulting method has comparable computational costs to FWI, since it is based on a objective functional whose gradient computation involves twice as many physical simulations required by FWI. Moreover, it is consistently more robust than FWI with respect to starting models and inexact modeling parameters not subject to inversion. Our approach represents a computationally efficient, robust, and ultimately feasible proposal for 3D seismic inversion.

Our proposal leverages current state-of-the-art in time-domain finitedifference solvers that ensures scalability on the hardware available to the oil-and-gas industry, or even to a wider audience thanks to cloud computing (Witte et al., 2020). We employ the domain-specific language Devito for automatic generation of highly optimized finite-difference $\mathrm{C}$ code (Luporini et al., 2020; Luporini et al., 2018; Louboutin et al., 2019). Here, we focus on acoustic modeling and pseudo-acoustic modeling with tilted transverse isotropy (TTI, Alkhalifah, 2000), established as a reasonable compromise between realistic physics and computational feasibility.

The computational and theoretical advantages of $\mathrm{WRI}^{*}$ will be demonstrated with extensive 2D experimentation. Despite the central claim of the paper being centered on 3D feasibility, realistic 3D problems are still beyond the economic resources realistically available to academic organizations, and will not be included in this work. Other fields relying on heavy computations, such as machine learning on large models, are plagued by similar problems. Therefore, we will present some preliminary results for a small $3 \mathrm{D}$ example. We stress that this is not due to a fundamental limitation of the method, and any institution that can afford the computations needed for FWI can also fruitfully employ WRI*.

In the rest of this paper, we discuss the computational and theoretical pitfalls of classical seismic inversion, with a general overview of the methods that deal with those issues. We review Lagrangian and reduced formulations for seismic inversion, followed by a more in-depth discussion on WRI. The main section is devoted to the reformulation of WRI object of this work: WRI*. Lastly, we present numerical experiments aiming at the comparison with FWI and conventional WRI. 


\section{Seismic inversion: computational and theoret- ical complexity}

Modern seismic inversion, such as FWI, is cast as an optimization problem endowed with partial differential equation constraints (Virieux and Operto, 2009). Given a suitable discretization of the physical quantities of interest, and the algebraic operators that govern their mutual relationship, the problem can be succinctly expressed as:

$$
\min _{\mathbf{m}, \mathbf{u}} \frac{1}{2}\|\mathbf{d}-R \mathbf{u}\|^{2} \quad \text { subject to } \quad A(\mathbf{m}) \mathbf{u}=\mathbf{q} .
$$

Data $\mathbf{d} \in \mathbb{R}^{N_{\mathrm{r}} \times N_{\mathrm{t}} \times N_{\mathrm{s}}}$ are recorded in time (where $N t$ denotes the number of time samples) at $N_{\mathrm{r}}$ receiver locations for different $N_{\mathrm{S}}$ source positions. Alternatively, after a temporal Fourier transform, $\mathbf{d} \in \mathbb{C}^{N_{\mathrm{r}} \times N_{\mathrm{f}} \times N_{\mathrm{s}}}$ for $N_{\mathrm{f}}$ frequencies. The variable $\mathbf{u} \in \mathbb{R}^{N \times N_{\mathrm{t}} \times N_{\mathrm{s}}}$ (or $\mathbf{u} \in \mathbb{C}^{N \times N_{\mathrm{f}} \times N_{\mathrm{s}}}$, in frequency domain) represents a wavefield- - e.g. the solution of the wave equation $A(\mathbf{m}) \mathbf{u}=\mathbf{q}$ - discretized on an $N$-dimensional space. The right-hand side $\mathbf{q}$ of the wave equation is typically a point source. In equation (1), the data are compared to the values of $\mathbf{u}$ interpolated at the receiver locations via the operator $R$, and the misfit is calculated via the least-squares norm $\|\cdot\|:=\|\cdot\|_{2}$. The wave operator $A(\mathbf{m})$ is parameterized by $\mathbf{m}$, which encapsulates the model unknowns of the problem. Throughout this paper, $\mathbf{m}$ represents squared slowness. Other physical parameters are included in the wave operator but kept fixed and will not be estimated.

In $3 \mathrm{D}$, the grid size grows as $N=\mathcal{O}\left(n^{3}\right)$ (in big $\mathcal{O}$ notation), with $n \approx 1000$ grid points in each direction for a typical industrial-scale problem. With dense source/receiver acquisition coverage, $N_{\mathrm{s}}=\mathcal{O}\left(n^{2}\right)$ and $N_{\mathrm{r}}=\mathcal{O}\left(n^{2}\right)$. Furthermore, when time-domain methods with explicit time-marching schemes are applied, $N_{\mathrm{t}}=\mathcal{O}(n)$ (realistically, $N_{\mathrm{t}} \approx 10 n$ ), due to stability conditions. In the frequency domain, we also often keep $N_{\mathrm{f}}=\mathcal{O}(n)$, albeit with much more favorable complexity constants than time domain (as suggested in Sirgue and Pratt, 2004). In this regime, the computational requirements are extremely challenging. For instance, wavefield storage grows as $\mathcal{O}\left(n^{6}\right)$, and cannot be attained for each source/time sample at once. Time complexity is largely dominated by the computation of the solutions of the wave equation. Currently, explicit time-domain methods are favored, as in the frequency domain the wave equation is represented by a large, sparse, non-definite linear system, and neither direct nor iterative methods scale as efficiently to 3D. Frequency domain becomes a palatable option only when a large high-performance computing system can store the matrix factorization (Wang et al., 2011), or abundant compute cores are available (Knibbe et al., 2016).

Despite these computational challenges, FWI is now viable for 3D problems of practical size, especially when the simulated physics is restricted to acoustics or tilted transversely isotropic (TTI) pseudo-acoustics (Alkhalifah, 2000; Grechka et al., 2004; Duveneck et al., 2008). The FWI objective functional is, however, highly multimodal and requires considerable problem-specific intervention to 
be consistently successful when gradient-based optimization is employed. In practice, FWI requires a kinematically correct starting guess to avoid cycle skipping. Local minima have been the subject of a great deal of research in the last 10 years, and many proposals tackling the problem have been advanced. The main lines of investigation involved:

- regularization techniques that penalize unrealistic models, e.g. via totalvariation minimization (Akcelik et al., 2002; Anagaw and Sacchi, 2012; Esser et al., 2018) or projection onto constraint sets (Peters and Herrmann, 2017; Peters et al., 2018);

- analysis of data misfit in a transformed domain (Shin and Cha, 2008, 2009; Bozdag et al., 2011) and/or application of preprocessing hierarchical strategies, where different levels of data preconditioning are setup and the associated problems solved sequentially, e.g. low- to high-frequency data filtering inversion (Bunks et al., 1995), or shallow- to deep-model inversion (Shipp and Singh, 2002);

- model extension, where the original problem is generalized to a higher dimensional space better suited for gradient-based optimization (Symes, 2008; van den Berg and Kleinman, 1997; Biondi and Almomin, 2014; van Leeuwen and Herrmann, 2013; Wang et al., 2016; Wang and Herrmann, 2017b; Aghamiry et al., 2019; Warner and Guasch, 2016; Guasch et al., 2019);

- special objective functionals more amenable to local-search optimization, e.g. based on traveltime-based misfit (Luo and Schuster, 1991; van Leeuwen and Mulder, 2010), or optimal transport via the Wasserstein distance (Métivier et al., 2016; Yang et al., 2018; Sun and Alkhalifah, 2019).

In practice, most of the inversion techniques typically benefit from a combination of these strategies.

Recently, many model extension methods have been proven successful in their empirical robustness to local minima, but are hampered by the computational costs associated to operating in higher dimension. A notable exception is adaptive waveform inversion (AWI, Warner and Guasch, 2016; Guasch et al., 2019), which consists of matching synthetic time traces to data by means of convolutional Wiener filters with arbitrary length. Since the computational overhead with respect to FWI is affordable, AWI can be applied to large problems. In this paper, however, we are not interested in establishing the relative merits of AWI with respect to other methods, and we will focus on developing a competing method based on WRI. While theoretically not immune to cycleskipping, as demonstrated in the recent work Symes (2020a) under simplifying 1-D assumptions, WRI is empirically more robust than FWI to local minima (as evidenced by a wealth of publications, starting from its original inception in van Leeuwen and Herrmann, 2013, to this very paper). Moreover, as suggested in Symes (2020a), a relatively straightforward modification of the WRI objective (based on a specific weighting of the equation error misfit) is already proven to be effective, albeit under the same simplifying conditions. Note that these 
modifications of WRI are readily implemented in this treatise. Despite these advantages, WRI in its basic formulation (van Leeuwen and Herrmann, 2013) is not amenable to 3D. Indeed, it involves a relaxed version of the wave equation that does not allow for a straightforward implementation of time-marching schemes and is practically limited to 2D problems (see, however, Peters and Herrmann, 2019, for some advances using frequency-domain 3D solvers). Alternative characterizations of the original WRI formulation, as offered in Huang et al. (2017), Huang et al. (2018), or Aghamiry et al. (2019), suffer from similar computational issues and do not scale efficiently to 3D. Note that the approaches of Huang et al. (2017), Huang et al. (2018) can be cast in the time domain, but still require expensive iterative solvers for the state variable, incurring the same computational bottleneck of WRI, or even FWI when formulated in the frequency domain. The main goal of this paper is to provide a reformulation of WRI that circumvents this limitation.

\section{Reduced, full-space, and penalty formulations}

In this section, we lay out the groundwork for WRI and our proposal WRI* by illustrating different ways to deal with the problem formulated in equation (1). Classical FWI consists of imposing the constraint $\mathbf{u}=A(\mathbf{m})^{-1} \mathbf{q}$ explicitly (from which the moniker of reduced formulation stems). Equation (1) can be equivalently rewritten as:

$$
\begin{aligned}
& \min _{\mathbf{m}} \mathcal{J}_{\mathrm{FWI}}(\mathbf{m}), \\
& \mathcal{J}_{\mathrm{FWI}}(\mathbf{m})=\frac{1}{2}\left\|\mathbf{d}-R A(\mathbf{m})^{-1} \mathbf{q}\right\|^{2} .
\end{aligned}
$$

Regularization can be enforced via constraints or extra penalty terms. There are several advantages to this formulation that makes large-scale deployment possible with the computational capabilities that are currently available. The objective functional $\mathcal{J}_{\mathrm{FWI}}$ is defined by a summation of the data misfit terms over individual sources. Therefore, in the evaluation of $\mathcal{J}_{\mathrm{FWI}}$ and its gradient calculation, the solution wavefields of forward and adjoint problems pertaining to a source can be discarded after use, thus limiting the memory overhead. In time domain, checkpointing techniques also reduce memory complexity (Symes, 2007). Alternatively, one could combine modeling in the time domain and the on-the-fly Fourier transform to obtain an estimate of the gradient by crosscorrelating the forward and backpropagated wavefields in the Fourier domain (Sirgue et al., 2008; Witte et al., 2019b). Although the reduced formulation leads to a scalable approach by making use of relatively frugal time-domain modeling, it is particularly prone to local minima, and necessitates a kinematically adequate starting guess for $\mathbf{m}$, ideally matching the data traveltimes within half a period (Beydoun and Tarantola, 1988).

Another classical setup, sometimes referred to as the full-space method, is by means of the Lagrangian function associated to equation (1) and the related 
saddle point problem (Haber et al., 2000):

$$
\begin{aligned}
& \max _{\mathbf{v}} \min _{\mathbf{m}, \mathbf{u}} \mathcal{L}_{\text {weq }}(\mathbf{m}, \mathbf{u}, \mathbf{v}), \\
& \mathcal{L}_{\text {weq }}(\mathbf{m}, \mathbf{u}, \mathbf{v})=\frac{1}{2}\|\mathbf{d}-R \mathbf{u}\|^{2}+\langle\mathbf{v}, \mathbf{q}-A(\mathbf{m}) \mathbf{u}\rangle,
\end{aligned}
$$

where $\langle\cdot, \cdot\rangle$ is the least-squares inner product. The multipliers $\mathbf{v}$ belong to a linear space with the same dimension as the wavefield $\mathbf{u}$ space. When memory usage is not of concern, we can carry out joint optimization over $\mathbf{m}, \mathbf{u}$ and $\mathbf{v}$. Note that the evaluation and gradient computation of the Lagrangian do not require the solution of the wave equation. Moreover, the Hessian of the Lagrangian is sparse and can be evaluated cheaply, making second-order methods affordable. However, in spite of these advantages, storing $\mathbf{u}$ and $\mathbf{v}$ in memory is not viable.

Penalty formulations are situated in between the full space method and FWI, and seek to enforce the wave equation weakly as a regularization term. By fixing the multiplier $\mathbf{v}=\lambda^{2}(\mathbf{q}-A(\mathbf{m}) \mathbf{u})$ in equation (3), we obtain the problem:

$$
\begin{aligned}
& \min _{\mathbf{m}, \mathbf{u}} \mathcal{J}_{\text {pen }}(\mathbf{m}, \mathbf{u}), \\
& \mathcal{J}_{\text {pen }}(\mathbf{m}, \mathbf{u})=\frac{1}{2}\|\mathbf{d}-R \mathbf{u}\|^{2}+\frac{\lambda^{2}}{2}\|\mathbf{q}-A(\mathbf{m}) \mathbf{u}\|^{2} .
\end{aligned}
$$

Here, $\lambda$ is a trade-off parameter between data misfit and wave equation error. Note that, with $\lambda \rightarrow \infty$, the method becomes equivalent to FWI. Similarly to the full space method, we cannot afford the storage of all the unknowns $\mathbf{u}$, and need to resort to the WRI approach described in the next section.

\section{$5 \quad$ Wavefield reconstruction inversion}

Within the penalty method framework in equation (4), van Leeuwen and Herrmann (2013) apply the variable projection scheme by defining $\overline{\mathbf{u}}(\mathbf{m}):=$ $\arg \min _{\mathbf{u}} \mathcal{J}_{\text {pen }}(\mathbf{m}, \mathbf{u})$ (Golub and Pereyra, 2003). This procedure yields the WRI method, whose reduced objective, function of only model variables $\mathbf{m}$, is:

$$
\begin{aligned}
& \min _{\mathbf{m}} \mathcal{J}_{\mathrm{WRI}}(\mathbf{m}), \\
& \mathcal{J}_{\mathrm{WRI}}(\mathbf{m})=\frac{1}{2}\|\mathbf{d}-R \overline{\mathbf{u}}(\mathbf{m})\|^{2}+\frac{\lambda^{2}}{2}\|\mathbf{q}-A(\mathbf{m}) \overline{\mathbf{u}}(\mathbf{m})\|^{2},
\end{aligned}
$$

where $\overline{\mathbf{u}}$ is the solution of the augmented wave equation:

$$
\left(\begin{array}{c}
R \\
\lambda A(\mathbf{m})
\end{array}\right) \overline{\mathbf{u}}(\mathbf{m})=\left(\begin{array}{c}
\mathbf{d} \\
\lambda \mathbf{q}
\end{array}\right)
$$

to be solved in least-squares sense. Equivalently,

$$
\left(R^{*} R+\lambda^{2} A(\mathbf{m})^{*} A(\mathbf{m})\right) \overline{\mathbf{u}}(\mathbf{m})=R^{*} \mathbf{d}+\lambda^{2} A(\mathbf{m})^{*} \mathbf{q} .
$$


Note that, by virtue of variable projection, $\nabla_{\mathbf{u}} \mathcal{J}_{\text {pen }}(\mathbf{m}, \overline{\mathbf{u}}(\mathbf{m}))=\mathbf{0}$, and

$$
\nabla_{\mathbf{m}} \mathcal{J}_{\text {WRI }}(\mathbf{m})=\nabla_{\mathbf{m}} \mathcal{J}_{\text {pen }}(\mathbf{m}, \overline{\mathbf{u}}(\mathbf{m})) .
$$

As in the reduced approach, WRI avoids simultaneous wavefield storage for each source (by computing and discarding). Empirical indications (including this paper) show that WRI is more robust than FWI with respect to local minima. Furthermore, WRI delivers consistently superior salt imaging (especially when combined with regularization, Esser et al., 2018; Peters and Herrmann, 2017; da Silva and Yao, 2017). Alas, a major drawback of WRI is that the augmented wave equation (6) cannot be trivially solved in neither frequency nor time domain when the problem is sizable.

Different reformulations of WRI have been proposed over the years that seek to overcome some of the computational limitations just described. In the following, we discuss some technical aspects related to the extended-source reformulation of WRI, object of the Wang et al. (2016) and Huang et al. (2018) studies.

Wang et al. (2016) reformulate WRI in terms of extended source variables rather than wavefields $\mathbf{u}$, via the change of variables $\mathbf{q}_{\text {ext }}:=A(\mathbf{m}) \mathbf{u}$. Rewriting equation (4) in terms of $\mathbf{q}_{\text {ext }}$, we obtain:

$$
\mathcal{J}_{\text {pen }}^{q}\left(\mathbf{m}, \mathbf{q}_{\text {ext }}\right)=\frac{1}{2}\left\|\mathbf{d}-F(\mathbf{m}) \mathbf{q}_{\mathrm{ext}}\right\|^{2}+\frac{\lambda^{2}}{2}\left\|\mathbf{q}-\mathbf{q}_{\mathrm{ext}}\right\|^{2} .
$$

where the forward operator is defined by

$$
F(\mathbf{m})=R A(\mathbf{m})^{-1} .
$$

Following the WRI approach, the extended source variables might be solved via variable projection, e.g. $\left(F(\mathbf{m})^{*} F(\mathbf{m})+\lambda^{2} I\right) \overline{\mathbf{q}}_{\mathrm{ext}}(\mathbf{m})=F(\mathbf{m})^{*} \mathbf{d}+\lambda^{2} \mathbf{q}$. To avoid the need for solving this linear system, the following approximation is introduced:

$$
\overline{\mathbf{q}}_{\mathrm{ext}}(\mathbf{m}) \approx \tilde{\mathbf{q}}_{\mathrm{ext}}(\mathbf{m})=\mathbf{q}+\frac{1}{\lambda^{2}} F(\mathbf{m})^{*} \mathbf{r}(\mathbf{m}), \quad \mathbf{r}(\mathbf{m})=\mathbf{d}-F(\mathbf{m}) \mathbf{q} .
$$

Here, $\mathbf{r}(\mathbf{m})$ is the data misfit residual for the model $\mathbf{m}$. The approximation in equation (11) can be regarded as the first iteration of a Krylov subspace solver applied to the system $F(\mathbf{m})^{*} F(\mathbf{m})+\lambda^{2} I$, and its accuracy increases as $\lambda \rightarrow \infty$ (which reveals the weakness of the approximation, since for $\lambda \rightarrow \infty$ WRI reverts to FWI). The computations related to equation (11) involve standard wave equation solvers, even in time domain, and are fit to realistic problems. A reduced objective is then introduced:

$$
\widetilde{\mathcal{J}}_{\mathrm{WRI}}^{q}(\mathbf{m})=\frac{1}{2}\left\|\mathbf{d}-F(\mathbf{m}) \tilde{\mathbf{q}}_{\mathrm{ext}}(\mathbf{m})\right\|^{2}+\frac{\lambda^{2}}{2}\left\|\mathbf{q}-\tilde{\mathbf{q}}_{\text {ext }}(\mathbf{m})\right\|^{2} .
$$

Since the extended source variable is only approximated, here, the computation of the gradient of $\widetilde{\mathcal{J}}_{\text {WRI }}^{q}$ is not trivial, and Wang et al. (2016) resort to the estimation:

$$
\nabla_{\mathbf{m}} \widetilde{\mathcal{J}}_{\mathrm{WRI}}^{q}(\mathbf{m}) \approx \nabla_{\mathbf{m}} \mathcal{J}_{\mathrm{pen}}^{q}\left(\mathbf{m}, \tilde{\mathbf{q}}_{\mathrm{ext}}(\mathbf{m})\right)
$$


Note that the dependence of $\widetilde{\mathbf{q}}_{\text {ext }}(\mathbf{m})$ with respect to $\mathbf{m}$ is not properly taken into account. The overall approach can indeed tackle large scale inversion, but is defective in two ways: the extended source approximation in equation (11) can be scaled badly and gradient-based optimization can suffer from the inaccurate gradient calculations involved in equation (13).

Volume source-based extended waveform inversion, by Huang et al. (2018), closely follow the extended source reformulation of Wang et al. (2016), although via a slightly different change of variable formula $\mathbf{q}_{\mathrm{ext}}:=\mathbf{q}-A(\mathbf{m}) \mathbf{u}$. For simplicity, we will discuss the work of Huang et al. (2018) with the extended source definition given by Wang et al. (2016). Contrary to Wang et al. (2016), here the augmented unknowns are solved via variable projection, and, analogously to WRI, a reduced objective can be defined only in terms of model variables $\mathbf{m}$ (therefore without suffering from the gradient approximation introduced in equation 13). One of the central contributions of Huang et al. (2018) is to refine the regularization term in (9) with prior knowledge about the source location $\mathbf{x}_{s}$, e.g. via the weighted penalty

$$
\mathcal{J}_{\text {pen }}^{q, \Sigma}\left(\mathbf{m}, \mathbf{q}_{\mathrm{ext}}\right)=\frac{1}{2}\left\|\mathbf{d}-F(\mathbf{m}) \mathbf{q}_{\mathrm{ext}}\right\|^{2}+\frac{\lambda^{2}}{2}\left\|\mathbf{q}-\mathbf{q}_{\mathrm{ext}}\right\|_{\Sigma}^{2},
$$

defined by the weighted least-squares norm $\left\|\mathbf{q}_{\text {ext }}\right\|_{\Sigma}:=\sqrt{\left\langle\Sigma^{-1} \mathbf{q}_{\text {ext }}, \mathbf{q}_{\text {ext }}\right\rangle}$. The weighting matrix $\Sigma^{-1}$ is chosen to penalize the contribution of the extended source located far from the physical source position, by imposing a diagonal structure $\Sigma^{-1}=\operatorname{diag}(\mathbf{w})$ corresponding to the spatial function:

$$
\mathbf{w}(\mathbf{x})=\left|\mathbf{x}-\mathbf{x}_{\mathbf{s}}\right| .
$$

The relevance of the weighted regularization on suppressing local minima has been recently discussed in Symes (2020b) and Symes (2020a), where conventional WRI with no weighting has been shown to exhibit the same local minima of FWI for some 1-D examples.

The extended source variables are functions of both space and time, and cannot be feasibly kept in memory all at once. Therefore, Huang et al. (2018) propose to restrict those unknowns to space only. While volume source-based extended waveform inversion is memory efficient, variable projection requires iterative solutions of a linear system akin to equation (6), hence sharing the same computational bottleneck as WRI.

\section{A dual formulation for wavefield reconstruc- tion inversion: WRI*}

A way to overcome the computational hurdles associated to the augmented wave equation is suggested by considering equation (7). Multiplying both sides of this expression by the inverse of $A(\mathbf{m})^{*}$ and simple algebraic manipulations yields

$$
A(\mathbf{m}) \overline{\mathbf{u}}(\mathbf{m})=\mathbf{q}+\overline{\mathbf{q}}(\mathbf{m}), \quad A(\mathbf{m})^{*} \overline{\mathbf{q}}(\mathbf{m})=R^{*} \overline{\mathbf{y}}(\mathbf{m}),
$$


for some variable $\overline{\mathbf{y}}(\mathbf{m})$ that belongs to the data space (* denotes the adjoint operation). In equation (16), $\overline{\mathbf{y}}(\mathbf{m})$ is backpropagated to form an additional source contribution $\overline{\mathbf{q}}(\mathbf{m})$. This extended source has a time and spatial distribution and undergirds the idea of extended-source inversion by Wang et al. (2016) and Huang et al. (2017) (previously discussed). Moreover,

$$
\overline{\mathbf{y}}(\mathbf{m})=(\mathbf{d}-R \overline{\mathbf{u}}(\mathbf{m})) / \lambda^{2} .
$$

This identity highlights that $\overline{\mathbf{y}}(\mathbf{m})$ is proportional to the data residual of the augmented wavefield defined in equation (6). It can be equivalently cast as the solution of the linear system:

$$
\left[\lambda^{2} I+F(\mathbf{m}) F(\mathbf{m})^{*}\right] \overline{\mathbf{y}}(\mathbf{m})=\mathbf{r}(\mathbf{m}) .
$$

$F(\mathbf{m})$ is the forward operator defined in equation (10), $I$ is the identity, and $\mathbf{r}(\mathbf{m})=\mathbf{d}-F(\mathbf{m}) \mathbf{q}$ is the data residual of the physical solution of the wave equation. The evaluation of the linear sytem in equation (18) involves solving the conventional wave equation (and adjoint thereof). If one could cheaply estimate $\overline{\mathbf{y}}(\mathbf{m})$, the augmented solution $\overline{\mathbf{u}}(\mathbf{m})$ would only require two conventional wave equation solutions.

These insights follow from first principles by starting with a denoising reformulation (Wang and Herrmann, 2017a) of equation (1),

$$
\min _{\mathbf{m}, \mathbf{u}} \frac{1}{2}\|\mathbf{q}-A(\mathbf{m}) \mathbf{u}\|^{2} \quad \text { s.t. } \quad\|\mathbf{d}-R \mathbf{u}\| \leq \epsilon,
$$

for a known noise level $\epsilon$. Notice how objective and constraints are here swapped with respect to the Lagrangian formulation for the full-space method in equation (3). The denoising problem in equation (19) is somewhat equivalent to the penalty formulation in equation (4), in the sense that for a fixed $\mathbf{m}$ and a given $\epsilon$, there exists a weight $\lambda$ such that the solutions of (4) and (19) are identical (and vice versa, Bjorck, 1996). The denoising formulation, however, has the advantage of a lower dimensional Lagrangian multiplier than the penalty counterpart, as described in the following sections.

\subsection{Lagrangian formulation for data misfit constraint}

We proceed similarly to the full-space method, by building the Lagrangian associated to the problem (19). In order to do so, we refer to the Fenchel duality theory (Rockafellar, 1970). The saddle-point problem associated to equation (19) takes the form

$$
\begin{aligned}
& \max _{\mathbf{y}} \min _{\mathbf{m}, \mathbf{u}} \mathcal{L}_{\text {dat }}(\mathbf{m}, \mathbf{u}, \mathbf{y}), \\
& \mathcal{L}_{\text {dat }}(\mathbf{m}, \mathbf{u}, \mathbf{y})=\frac{1}{2}\|\mathbf{q}-A(\mathbf{m}) \mathbf{u}\|^{2}+\langle\mathbf{y}, \mathbf{d}-R \mathbf{u}\rangle-\epsilon\|\mathbf{y}\|,
\end{aligned}
$$

where the slack variables $\mathbf{y}$ belong to a linear space with the same dimension as data. We can eliminate the wavefield variables $\mathbf{u}$ from equation (20) by applying 
the variable projection $\overline{\mathbf{u}}(\mathbf{m})=\arg \min _{\mathbf{u}} \mathcal{L}_{\text {dat }}(\mathbf{m}, \mathbf{u}, \mathbf{y})$ :

$$
A(\mathbf{m}) \overline{\mathbf{u}}(\mathbf{m})=\mathbf{q}+\overline{\mathbf{q}}(\mathbf{m}), \quad A(\mathbf{m})^{*} \overline{\mathbf{q}}(\mathbf{m})=R^{*} \mathbf{y},
$$

in a similar fashion to what was described in equation (16). The substitution $\mathcal{L}_{\mathrm{WRI}^{*}}(\mathbf{m}, \mathbf{y}):=\mathcal{L}_{\text {dat }}(\mathbf{m}, \overline{\mathbf{u}}(\mathbf{m}), \mathbf{y})$ yields the $\mathrm{WRI}^{*}$ problem

$$
\begin{aligned}
& \max _{\mathbf{y}} \min _{\mathbf{m}} \mathcal{L}_{\mathrm{WRI}^{*}}(\mathbf{m}, \mathbf{y}), \\
& \mathcal{L}_{\mathrm{WRI}^{*}}(\mathbf{m}, \mathbf{y})=-\frac{1}{2}\left\|F(\mathbf{m})^{*} \mathbf{y}\right\|^{2}+\langle\mathbf{y}, \mathbf{r}(\mathbf{m})\rangle-\epsilon\|\mathbf{y}\|,
\end{aligned}
$$

where the forward operator $F(\mathbf{m})=R A(\mathbf{m})^{-1}$ was introduced in equation (10), and $\mathbf{r}(\mathbf{m})=\mathbf{d}-F(\mathbf{m}) \mathbf{q}$ is the data residual for the model $\mathbf{m}$. In equation (22), optimizing over $\mathbf{y}$ means maximizing the correlation of $\mathbf{y}$ with the data residual $\mathbf{r}(\mathbf{m})$, where additional regularization terms penalize the magnitudes of $\mathbf{y}$ and the extended source $\overline{\mathbf{q}}(\mathbf{m})=F(\mathbf{m})^{*} \mathbf{y}$. Under this lens, the method bears some resemblance with techniques based on data cross-correlation thoroughly investigated in the past starting from the work of Luo and Schuster (1991).

Regarding the gradient calculation, we start by denoting with $J[\mathbf{m}, \mathbf{f}]=$ $\partial_{\mathbf{m}}(F(\mathbf{m}) \mathbf{f})$ the Jacobian of the mapping $\mathbf{m} \mapsto F(\mathbf{m}) \mathbf{f}$, for a general source $\mathbf{f}$ (we make here a notational distinction between the derivative of a vector-valued function, indicated by $\partial_{\mathbf{m}}$, and the derivative of a real-valued function, indicated by $\left.\nabla_{\mathbf{m}}\right)$. The action of the adjoint of $J[\mathbf{m}, \mathbf{f}]$ can be obtained by differentiating both sides of the identity $\langle\mathbf{y}, F(\mathbf{m}) \mathbf{f}\rangle=\left\langle F(\mathbf{m})^{*} \mathbf{y}, \mathbf{f}\right\rangle$, which gives $J[\mathbf{m}, \mathbf{f}]^{*} \mathbf{y}=$ $\partial_{\mathbf{m}}\left(F(\mathbf{m})^{*} \mathbf{y}\right)^{*} \mathbf{f}$. This promptly yields $\nabla_{\mathbf{m}} \frac{1}{2}\left\|F(\mathbf{m})^{*} \mathbf{y}\right\|^{2}=J\left[\mathbf{m}, F(\mathbf{m})^{*} \mathbf{y}\right]^{*} \mathbf{y}$ and $\nabla_{\mathbf{m}}\langle\mathbf{y}, \mathbf{r}(\mathbf{m})\rangle=-J[\mathbf{m}, \mathbf{q}]^{*} \mathbf{y}$. Therefore, we obtain

$$
\begin{gathered}
\nabla_{\mathbf{m}} \mathcal{L}_{\mathrm{WRI}^{*}}=-J\left[\mathbf{m}, \mathbf{q}+F(\mathbf{m})^{*} \mathbf{y}\right]^{*} \mathbf{y} \\
\nabla_{\mathbf{y}} \mathcal{L}_{\mathrm{WRI}^{*}}=\mathbf{d}-F(\mathbf{m})\left(\mathbf{q}+F(\mathbf{m})^{*} \mathbf{y}\right)-\epsilon \mathbf{y} /\|\mathbf{y}\| .
\end{gathered}
$$

The expression in equation (23) can be computed similarly to a conventional FWI gradient: $(i)$ compute $\overline{\mathbf{q}}(\mathbf{m})$ in equation (21) by backward propagation, (ii) compute $\overline{\mathbf{u}}(\mathbf{m})$ in equation (21) by forward propagation, and (iii) perform temporal cross-correlation of $\overline{\mathbf{q}}(\mathbf{m})$ and $\partial_{t t} \overline{\mathbf{u}}(\mathbf{m})$. The gradient with respect to $\mathbf{y}$ in equation (24) corresponds to the data residual of the augmented wavefield in equation (21), plus a relaxation term.

The fundamental gain in solving WRI* instead of WRI lies in the fact that the evaluation of the Lagrangian $\mathcal{L}_{\mathrm{WRI}^{*}}$ and its gradients only require solutions of a conventional wave equation, opening up the choice for time-domain solvers. The multipliers $\mathbf{y}$ can be stored in memory whenever $\mathbf{m}$ and $\mathbf{y}$ are jointly optimized. Alternatively, one might consider variable projection for $\mathbf{y}$. Both these approaches are computationally expensive, and in this paper we will rely on a simple approximation for $\mathbf{y}$, to be discussed in the next section. 


\subsection{Dual variable approximation}

Equating the gradient with respect to $\mathbf{y}$ to zero, in equation (24), provides a closed-form solution for the optimal $\overline{\mathbf{y}}(\mathbf{m})$, defined by the non-linear system:

$$
\epsilon \frac{\overline{\mathbf{y}}(\mathbf{m})}{\|\overline{\mathbf{y}}(\mathbf{m})\|}+F(\mathbf{m}) F(\mathbf{m})^{*} \overline{\mathbf{y}}(\mathbf{m})=\mathbf{r}(\mathbf{m})
$$

analogously to equation (18) for conventional WRI. Note that a dualization of the data misfit term in the penalty method in equation (4) (following, for example, Chambolle and Pock, 2011) would produce a Lagrangian

$$
\mathcal{L}_{\mathrm{WRI}^{*}, \lambda}(\mathbf{m}, \mathbf{y})=-\frac{1}{2}\left\|F(\mathbf{m})^{*} \mathbf{y}\right\|^{2}+\langle\mathbf{y}, \mathbf{r}(\mathbf{m})\rangle-\frac{\lambda^{2}}{2}\|\mathbf{y}\|^{2}
$$

quite similar to the Lagrangian in equation (22). Not surprisingly, the optimal $\overline{\mathbf{y}}_{\lambda}(\mathbf{m})$ for equation (26) solves the same linear system in equation (18) for WRI, and the reduced problem is equivalent to WRI, since $\mathcal{L}_{\mathrm{WRI}^{*}, \lambda}\left(\mathbf{m}, \overline{\mathbf{y}}_{\lambda}(\mathbf{m})\right)=$ $\mathcal{J}_{\text {WRI }}(\mathbf{m}) / \lambda^{2}$.

Equation (25) (or its linear variant 26), can be solved iteratively. However, each evaluation of equation (25) amounts to two wave equation solutions and many iterations may be required. As a result, we propose a cheap approximation for $\overline{\mathbf{y}}(\mathbf{m})$ corresponding to the scaled data residual $\overline{\mathbf{y}}(\mathbf{m}) \approx \tilde{\mathbf{y}}(\mathbf{m})=\alpha \mathbf{r}(\mathbf{m})$, with an unknown scaling factor $\alpha$. Given that equation (22) is (strictly) concave with respect to $\mathbf{y}$, the optimal scaling $\tilde{\alpha}(\mathbf{m})=\arg \max _{\alpha} \mathcal{L}_{\mathrm{WRI}^{*}}(\mathbf{m}, \alpha \mathbf{r}(\mathbf{m}))$ is uniquely solved by

$$
\begin{aligned}
& \tilde{y}=\tilde{\alpha}(\mathbf{m}) \mathbf{r}(\mathbf{m}), \\
& \tilde{\alpha}(\mathbf{m})= \begin{cases}\frac{\|\mathbf{r}(\mathbf{m})\|(\|\mathbf{r}(\mathbf{m})\|-\epsilon)}{\left\|F(\mathbf{m})^{*} \mathbf{r}(\mathbf{m})\right\|^{2}}, & \|\mathbf{r}(\mathbf{m})\| \geq \epsilon, \\
0, & \text { otherwise. }\end{cases}
\end{aligned}
$$

There are no significant extra costs associated with this computation, as it can be obtained as a byproduct of the calculations involved in the evaluation of the Lagrangian functional. The scaled residual as an approximation improves on the approach taken in Wang et al. (2016), previously discussed, thanks to the optimal scaling. More interestingly, owing to its simplicity, it allows analytical differentiation, as shown in the next section.

\subsection{A new objective based on the dual formulation}

The approximation for $\mathbf{y}$ introduced in equation (27) results in the reduced objective

$$
\widetilde{\mathcal{L}}_{\mathrm{WRI}^{*}}(\mathbf{m}):=\mathcal{L}_{\mathrm{WRI}^{*}}(\mathbf{m}, \tilde{\mathbf{y}}(\mathbf{m})),
$$

and its gradient with respect to $\mathbf{m}$ is

$$
\nabla_{\mathbf{m}} \widetilde{\mathcal{L}}_{\mathrm{WRI}^{*}}=\nabla_{\mathbf{m}} \mathcal{L}_{\mathrm{WRI}^{*}}(\mathbf{m}, \tilde{\mathbf{y}}(\mathbf{m}))-\tilde{\alpha} J[\mathbf{m}, \mathbf{q}]^{*} \nabla_{\mathbf{y}} \mathcal{L}_{\mathrm{WRI}^{*}}(\mathbf{m}, \tilde{\mathbf{y}}(\mathbf{m})),
$$


where $\nabla_{\mathbf{m}} \mathcal{L}_{\mathrm{WRI}^{*}}$ and $\nabla_{\mathbf{y}} \mathcal{L}_{\mathrm{WRI}^{*}}$ are given in equations (23) and (24). For simplicity, we will still refer to the reduced problem as WRI*. Note that the computation of equation (29) does not require the differentiation of $\tilde{\alpha}(\mathbf{m})$ with respect to $\mathbf{m}$, as its expression has been obtained from variable projection. Neglecting the second term in equation (29) (analogously to Wang et al., 2016) saves some computation but, as also observed in practice, it has a detrimental effect on the convergence of nonlinear optimization methods, such as l-BFGS (Nocedal and Wright, 2006). The evaluation and gradient calculation of the WRI* objective (28) is schematically reported in algorithm 1 , and can be used in combination with any nonlinear optimization solver.

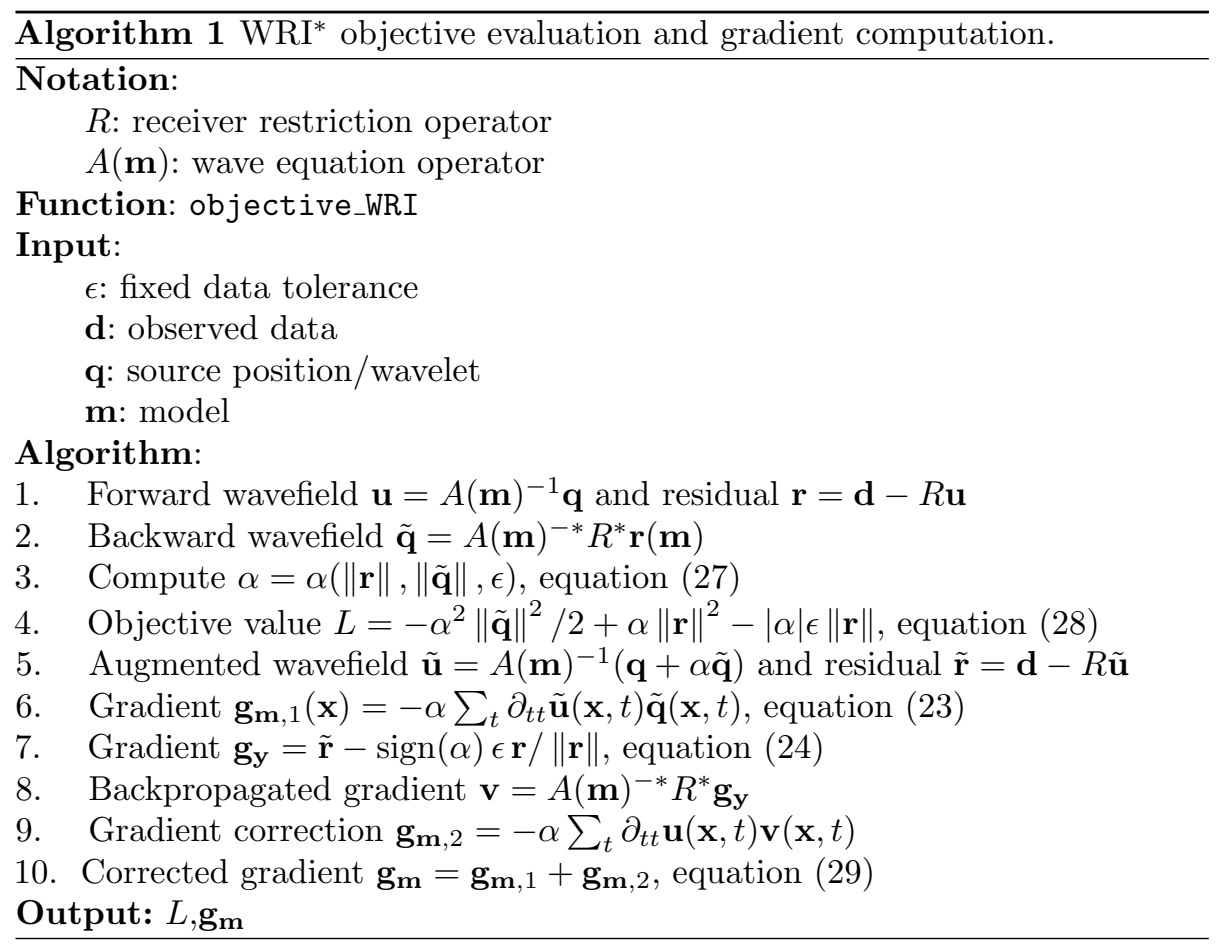

\subsection{Source-dependent metrics for the wave equation misfit}

In equation (19), we can exploit the knowledge about the location and distribution of the physical source $\mathbf{q}$, and design metrics for the wave equation misfit that are more suited for the problem at hand. As previously discussed, Huang et al. (2018) choose a weighted least-squares norm that penalizes functions with a support distributed far from the point source location. Some other recent work by Symes (2020a), demonstrates that WRI is susceptible to local minima when no source-weighting is applied. Here, we then select a penalty grid function $\mathbf{w}(\mathbf{x})=\sqrt{\left|\mathbf{x}-\mathbf{x}_{\mathbf{s}}\right|^{2}+h^{2}} / h$ dependent on a parameter $h$ tuned to be a fraction of a reference wavelength. With this modification, the Lagrangian in equation (22) 
becomes

$$
\mathcal{L}_{\mathrm{WRI}^{*}}(\mathbf{m}, \mathbf{y})=-\frac{1}{2}\left\|F(\mathbf{m})^{*} \mathbf{y}\right\|_{\Sigma^{-1}}^{2}+\langle\mathbf{y}, \mathbf{r}(\mathbf{m})\rangle-\epsilon\|\mathbf{y}\|
$$

and only minor corrections are needed for related gradient expressions and dual variable approximation. In the experiments in the next section, we tacitly assume this type of source-dependent weighting. Note that source weighting cannot be trivially incorporated in FWI, since it is based on the exact solution of the wave equation. Beside the theoretical considerations in Symes (2020a), source weighting is beneficial to enhance the resolution for portions of the model located far from the source position. We point out that the hyperparameter $h$ should be small enough to promote the focusing of the additional source term $F(\mathbf{m})^{*} \mathbf{y}$ in equation (30).

Other potentially interesting modifications of the WRI*objective in equation (22) involve the $\ell_{1}$ norm, $\|\cdot\|_{1}$, or weighted versions thereof, to measure the wave equation error. The basic rationale is to impose sparsity on the spatial distribution of the extended source. We refer to Sharan et al. (2018) for a microseismic application. Following Sharan et al. (2018), we further suggest the so-called elastic net regularization obtained by replacing the $\ell_{1}$ norm with a relaxation that involves the least-squares norm: $\|\cdot\|_{1}+\|\cdot\|_{2}^{2} /\left(2 \mu^{2}\right)$. This relaxation is key in obtaining an analytical expression for the dual objective, analogously to equation (22). In this paper, however, we defer the $\ell_{1}$ formulation to future work, and stick to the objective (22).

\subsection{Complexity of WRI*}

Compared to FWI, evaluating and differentiating (28) requires the computation of twice as many solutions of the wave equation, respectively. Despite the increased cost, this still compares favorably to conventional WRI, since we avoid the need to solve for an augmented wave equation and frequency domain solvers. Moreover, as it will be demonstrated with numerical examples, WRI* retains the robustness against local minima characteristic of classical WRI, despite the approximations discussed in the previous sections.

\subsection{Beyond the dual variable approximation}

The approximation for the dual variable introduced in equation (27) is motivated by computational reasons and the need for analytical expressions for the objective gradient, here available precisely because of its simple expression. Naturally, a more accurate approximation of the optimal solution $\overline{\mathbf{y}}(\mathbf{m})$ in equation (25) can be attained by iteratively solving the non-linear system in equation (25). If we denote by $\tilde{\mathbf{y}}^{n}(\mathbf{m})$ the estimate obtained after $n$ iterations of a given solver for the equation (25), we can define the corresponding reduced objective by $\widetilde{\mathcal{L}}_{\mathrm{WRI}^{*}}^{n}(\mathbf{m}):=\mathcal{L}_{\mathrm{WRI}^{*}}\left(\mathbf{m}, \tilde{\mathbf{y}}^{n}(\mathbf{m})\right)$, analogously to equation (28). Clearly, $\mathrm{WRI}^{*}$ corresponds to $n=1$. For $n>1$, differentiating $\widetilde{\mathcal{L}}_{\text {WRI* }}^{n}$ will likely require automatic differentiation (Griewank and Walther, 2008). Note that one 
can neglect the dependency of the augmented variable with respect to $\mathbf{m}$ with adaptively accurate solves, as demonstrated in van Leeuwen and Herrmann (2014), so that an exact gradient computation is not strictly needed for convergence. Recently, however, it has been shown that rigorous gradient calculations lead to "super-efficient" estimators for the optimal gradient (Ablin et al., 2020). Investigating this line of research is not within the scope of this paper and is left to future studies.

\section{Results}

We test the capabilities of the WRI* inversion method with synthetic examples.

We start by comparing WRI* with classical WRI. We remind that WRI* and WRI are equivalent only when the dual variable is solved exactly, as in equation (25) (with the caveat described in that section). In other words, we will test the effect of the dual variable approximation proposed in equation (27). Note that this set of experiments will be carried out in the frequency domain, since traditional WRI is therein framed more conveniently from a computational point of view.

Other experiments mainly aim at the empirical demonstrations of the following claims:

- $\mathrm{WRI}^{*}$ is more robust than FWI with respect to local minima;

- WRI* is more robust than FWI with respect to modeling inaccuracies.

With modeling inaccuracies, we refer to erroneous assumptions about some of the physical parameters that will be kept fixed during inversion, in the context of TTI modeling (tilted transverse isotropy) and inversion.

Finally, we present a small-sized 3D problem with a comparison of initial gradients of WRI* and FWI, as a testament for potential full-sized applications.

\subsection{WRI vs WRI*}

The aim of this experiment is a qualitative assessment of the dependency of the WRI* scheme on the hyperparameter $\epsilon$, appearing in equation (19) (which denotes a given data noise tolerance), and the comparison with WRI. We emphasize that one of the main differences between WRI* and classical WRI is a suboptimal — but computationally convenient - approximation of the slack variables y described in (27) (while the optimal y makes WRI* and WRI substantially equivalent). The scope of the example described in this section is therefore twofold:

- determine the effect of $\epsilon$ on the WRI* inversion result;

- determine the effect of the dual variable approximation in equation (27) by comparing WRI* with traditional WRI (based on the optimal solution for $\mathbf{y}$ via variable projection). 


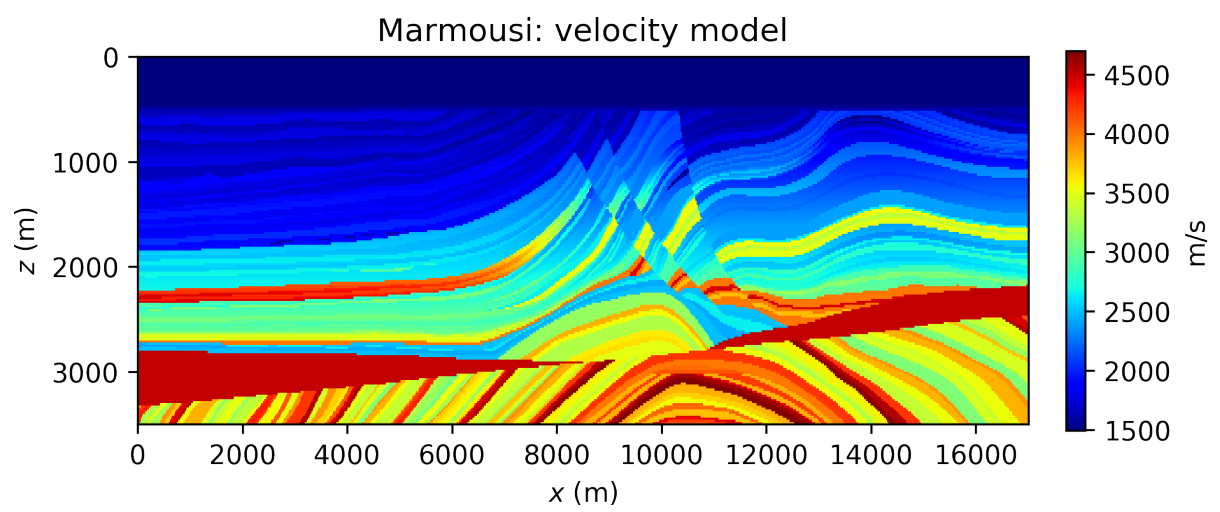

(a)

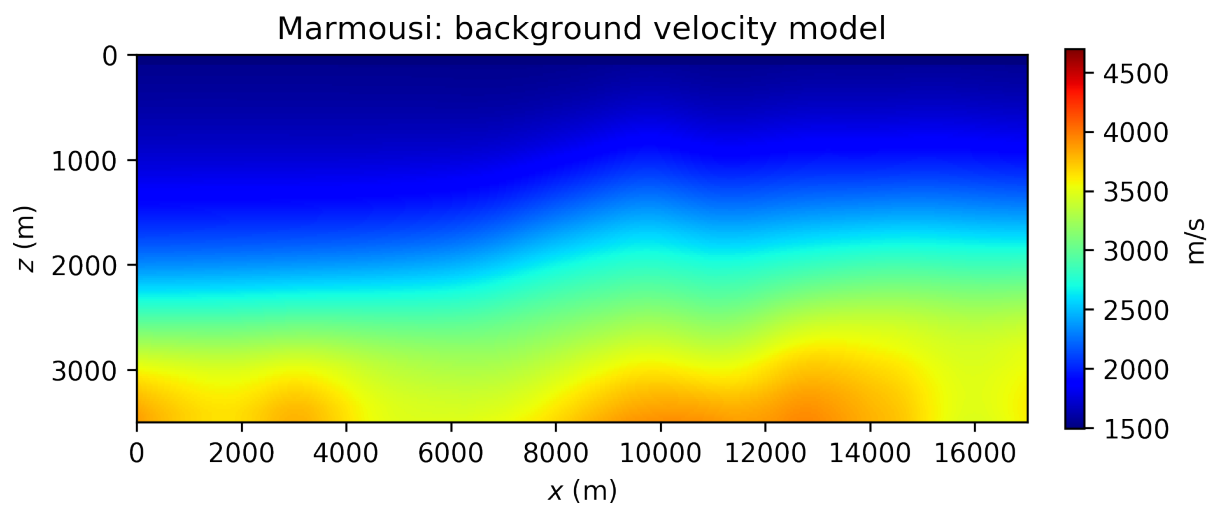

(b)

Figure 1: Marmousi model: (a) true velocity model, (b) background model. We invert single-frequency data components with a multiscale approach (Bunks et al., 1995) from low to high frequencies, and run 10 iterations of the l-BFGS optimizer per frequency. We compare FWI, WRI* with different values for $\epsilon$, and conventional WRI (with a fixed choice for the weighting parameter $\lambda$ in equation 26).

The classical WRI formulation is defined by the objective functional in equation (26), where $\mathbf{y}$ is obtained by (18). Note that the experiment of this section will be carried out in the frequency domain, where WRI is more conveniently formulated.

We consider the well-known Marmousi model, presented in Figure 1a. Data are directly generated in the frequency domain with components ranging from $3 \mathrm{~Hz}$ to $14 \mathrm{~Hz}$, with no additional noise (Figure \#fig:marmdata). We set 100 sources evenly spaced and receivers densely sampled along the surface.

Comparing the results in Figure 2, it is apparent that FWI converges to a local minimum, while both $\mathrm{WRI}^{*}$ and conventional WRI produce plausible models. 


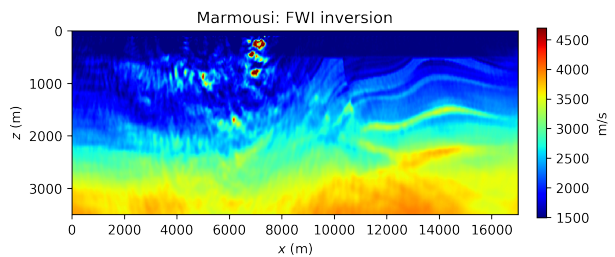

(a)

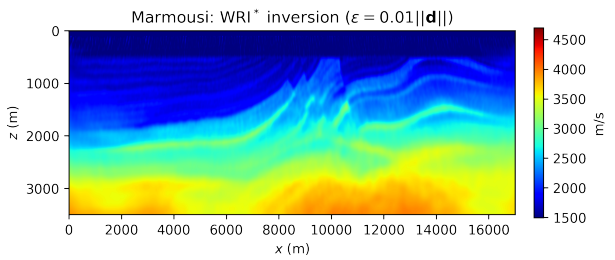

(b)

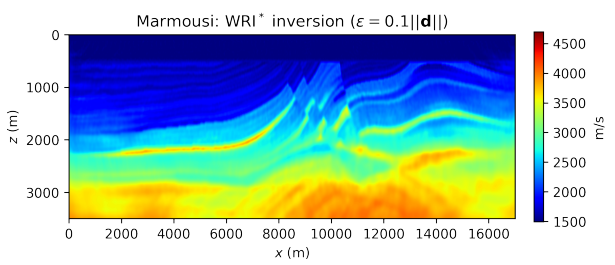

(c)

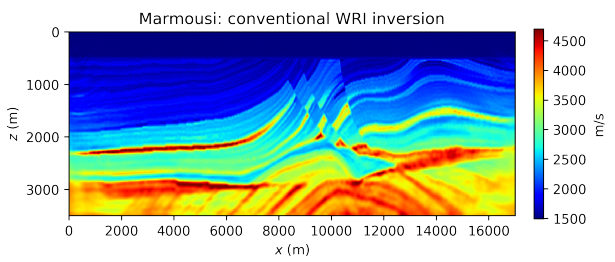

(d)

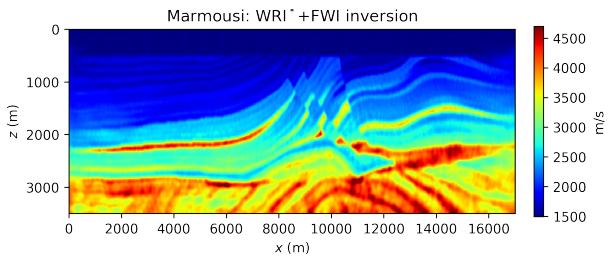

(e)

Figure 2: Marmousi inversion result for: (a) FWI, (b) WRI* $(\epsilon=0.01\|\mathbf{d}\|)$, (c) $\mathrm{WRI}^{*}(\epsilon=0.1\|\mathbf{d}\|)$, (d) conventional WRI, (e) sequential inversion: WRI* $(\epsilon=0.01\|\mathbf{d}\|)$ followed by FWI with Gauss-Newton preconditioning. 
WRI* does not achieve the same resolution of the WRI inversion, although we observe that increasing values for $\epsilon$ translate to higher model resolution (cf. Figures $2 \mathrm{~b}$ and $2 \mathrm{c}$ ). This behavior can be understood in the following qualitative terms: lower values for $\epsilon$ promote a wavefield solution that fits data rather than the wave equation (see, for instance, equation 19). Relaxing the wave equation is beneficial to avoid local minima, as advocated in the original WRI method, but it translates to inaccurate model resolution. Note that, in WRI, the $\lambda$ parameter appearing in equation (5) plays the same role as $\epsilon$.

Despite the similarities between WRI and WRI*, the WRI result is generally less affected by the choice of the relaxation parameter $\lambda$ compared to WRI* with $\epsilon$ (see Figure 2d). This behavior is dictated by the sub-optimal dual variable estimate in WRI*. In our experience, however, WRI* conserves the same ability of WRI to circumvent local minima, and the gap between the two methods can be reduced by either quasi-Newton preconditioning or devising a sequential WRI*-FWI scheme where the WRI* result is sharpened by FWI (which will also help in saving computations once local minima are avoided). This approach is also suggested in Guasch et al. (2019). An example of the joint WRI*-FWI is depicted in Figure 2e, highlighting a qualitatively similar result to conventional WRI in Figure 2d. Note that this hybrid scheme might lose the benefit of WRI* in dealing with inexact modeling assumptions. In that case, we might replace the least-squares misfit in FWI with more robust objectives as proposed in Herrmann et al. (2013).

Finally, in Figure 3 we compare reverse time migration (RTM, Baysal et al., 1983) obtained from different inversion results. For this purpose, high-frequency time-domain synthetic data is generated with the same source-receiver acquisition parameters used in the inversion phase, but with a Ricker source wavelet of 20 $\mathrm{Hz}$ peak frequency. The comparison is made with RTM obtained from the initial background, conventional WRI, and hybrid WRI*/FWI inversion results. All these results improve on the starting RTM image, with higher resolution, more continuous, and more accurately positioned reflectors (especially in the central portion of the model). The migration quality clearly improves with the resolution of the inverted model, with deeper reflectors better resolved by conventional WRI and hybrid WRI*/FWI strategies.

\subsection{FWI vs WRI*}

\subsubsection{Low-velocity lens}

This numerical experiment is geared towards a direct comparison of WRI* and FWI, and demonstrates the relative robustness of WRI* against spurious modes. We consider the Gaussian lens model presented in (Huang et al., 2018), here reproduced in Figure 4. The problem consists of imaging a low-velocity anomaly of Gaussian shape from transmission data, starting with an homogeneous model. Contrary to the previous example, we run this experiment in time domain. The synthetics are generated with a Ricker source wavelet of $10 \mathrm{~Hz}$ peak frequency (corresponding to a spatial wavelength of $200 \mathrm{~m}$ ), with no artificial noise added. 


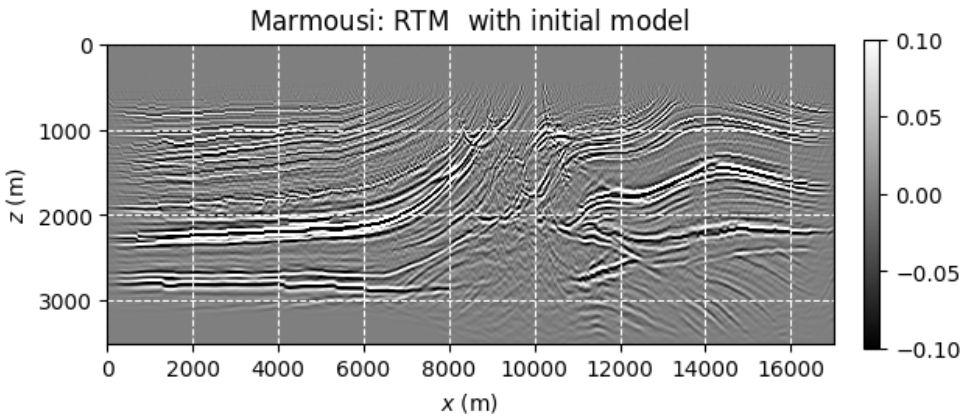

(a)

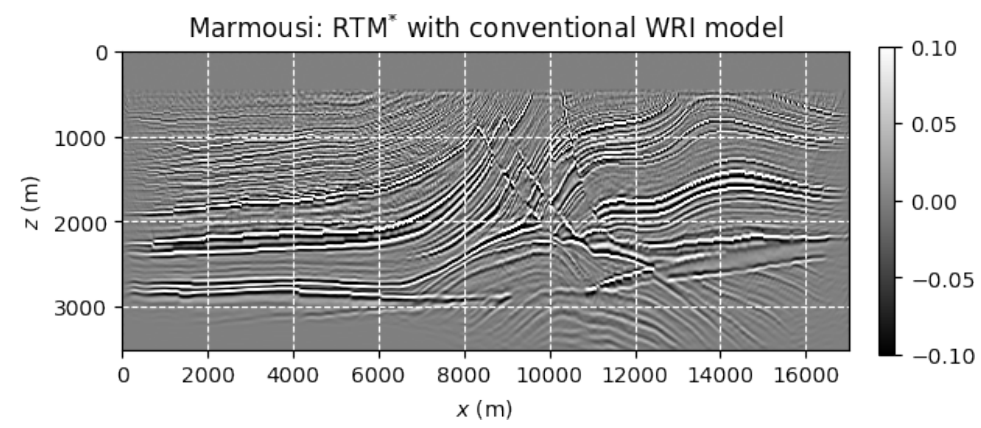

(b)

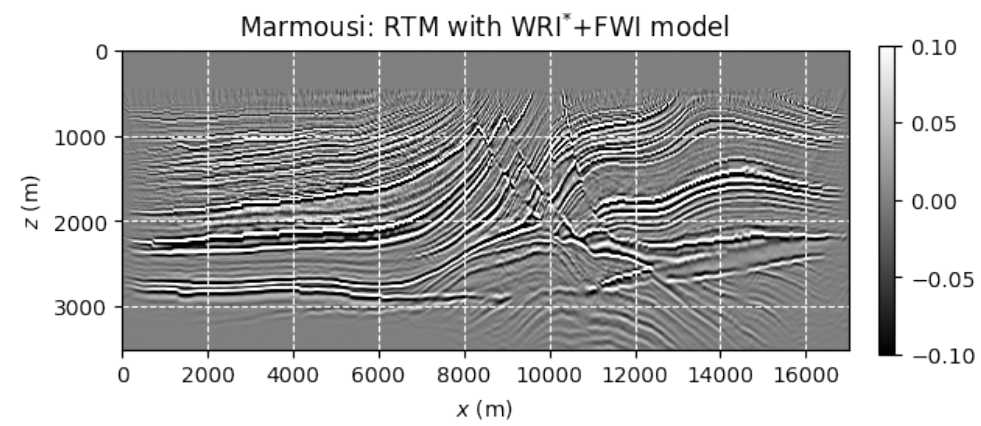

(c)

Figure 3: Reverse time migrations (normalized for display purposes) for the Marmousi model obtained from the following velocity models: (a) starting model (Figure 1b), (b) conventional WRI (Figure 2d), (c) combined WRI*/FWI strategy (Figure 2e). A grid is overlayed in white to aid the comparison. 


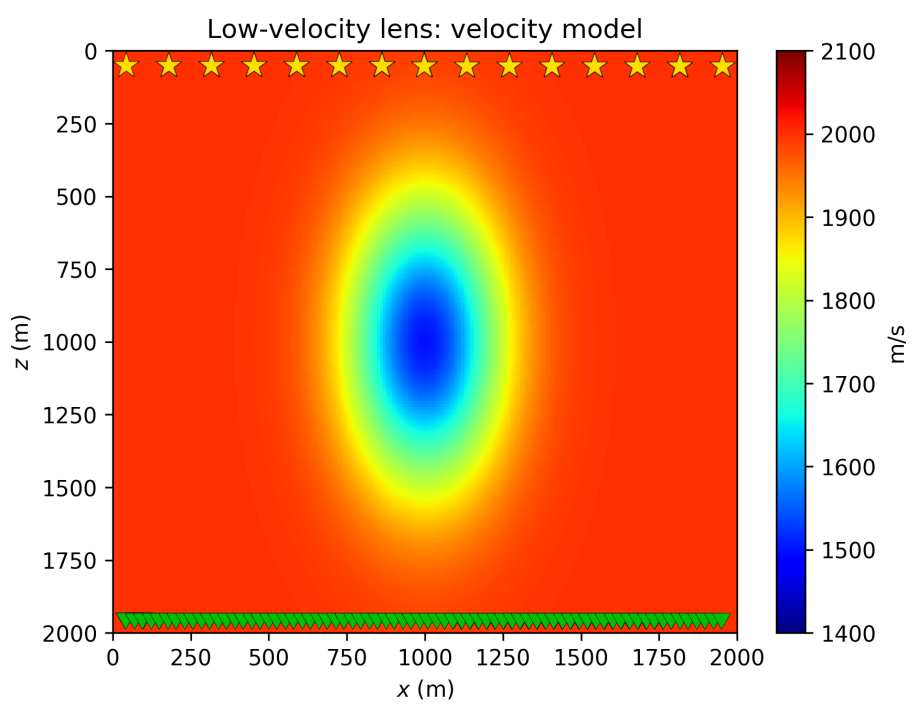

Figure 4: Low-velocity lens model. Sources are indicated by stars, and receivers by triangles. In this experiment, we run 20 iterations of the l-BFGS optimizer (Nocedal and Wright, 2006). Due to cycle skipping, FWI converges to a local minimum, shown in Figure 5a. We stress the fact both WRI or WRI* need be used in combination with source weighting (equation 30) to converge to the solution (as also documented in Huang et al., 2018; Symes, 2020a,b). The WRI* result (obtained with data tolerance $\epsilon=0$ ) is depicted in Figure 5b. We observe that WRI* is clearly able to reconstruct the anomaly and is not stuck to a local minimum.

We model 15 sources, and gather data with approximately 200 receivers (evenly spaced). The low-velocity zone generates triplications and eventually causing cycle skipping.

\subsubsection{BG Compass}

We focus now not only on how WRI* and FWI handle cycle skipping, but also how inaccurate assumptions about the underlying physical model of the data reflect on the inversion result. For example, what is the effect of not accounting for the correct anisotropic model when data come from anisotropic modeling? We show that WRI*, being based on a relaxation of the wave equation, has the potential to mitigate erroneous assumptions, while FWI will be more heavily affected. We therefore compare the results of WRI* and FWI on the BG Compass model according to two distinct scenarios:

- perfect modeling assumptions: both given data and modeled synthetics are obtained from the same physical model (in particular, acoustic modeling). The inversion parameter is the squared slowness; 


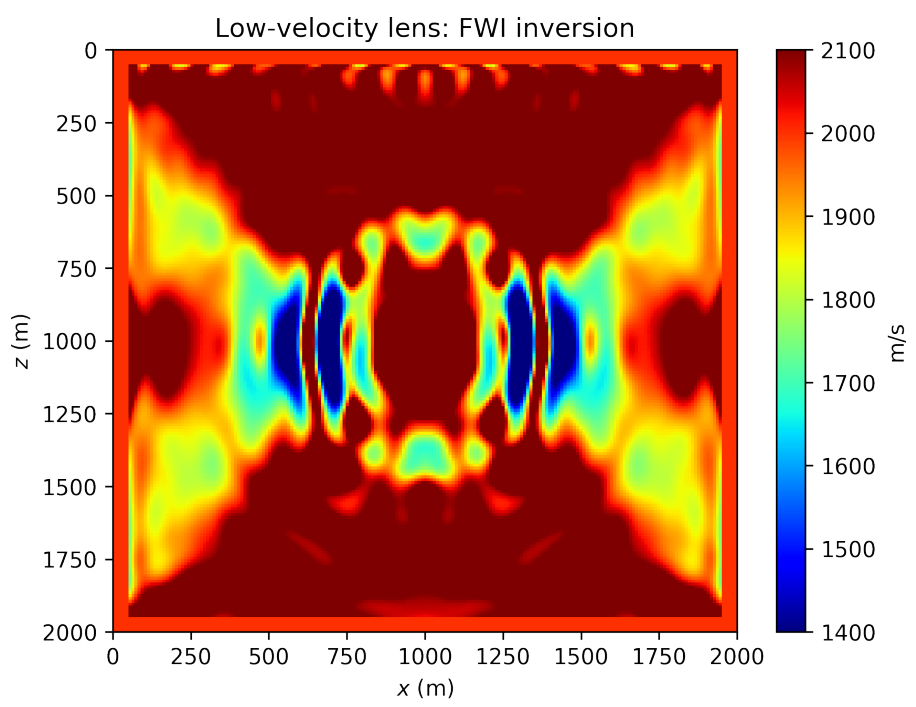

(a)

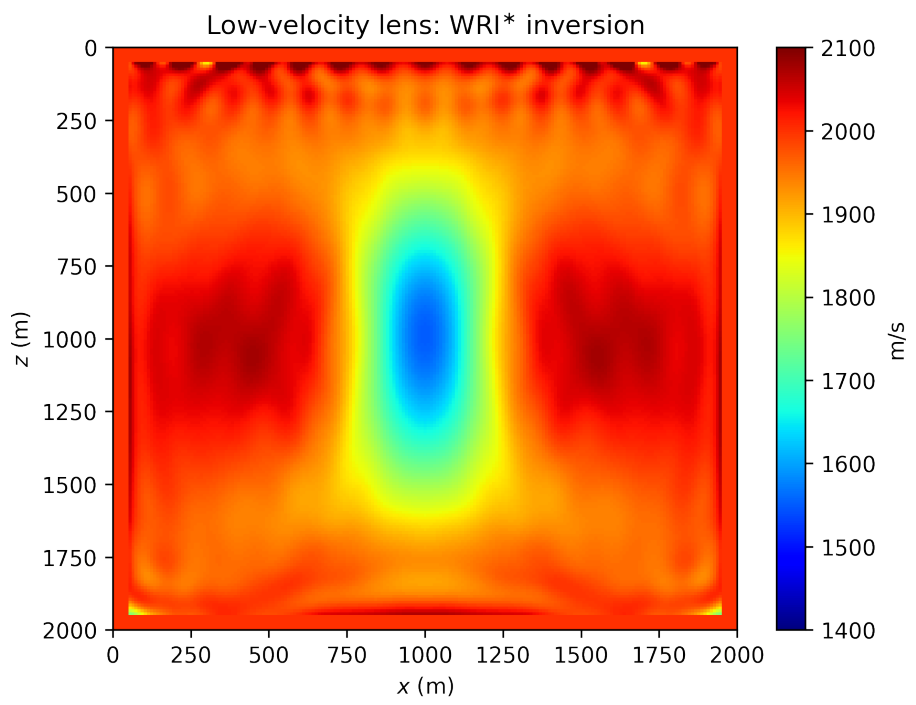

(b)

Figure 5: Inversion results for the Gaussian lens problem: (a) FWI inversion result, (b) WRI* inversion result.

- inaccurate modeling assumptions: given data are produced with a certain TTI model (tilted transverse isotropy), while synthetics are modeled with an inaccurate TTI model. All the TTI parameters but the squared slowness are kept fixed during inversion.

As in the previous example, this set of experiments is carried out in time 
domain. The data tolerance (defined in equation 19) will be set to $\epsilon=0$ for all the following experiments.

Inversion with correct modeling assumptions The acoustic (constant density) BG Compass model, here considered, is representative of some of the complexities encounter in the field, and leads to a notoriously difficult problem for FWI due to the velocity kickback approximately located at the depth of 1 Km (see Figure 6), delaying turning waves traveling back to the surface. We emphasize that the BG Compass here considered is made larger than the original version to exacerbate these challenges.

We model 51 sources with a Ricker wavelet (peak frequency $10 \mathrm{~Hz}$ ), and record data with dense receiver coverage (sampling rate according to the grid spacing), without additional noise. Note that the frequency components below 5 $\mathrm{Hz}$ in the data are filtered out and not used during inversion. The difficulties associated with the BG Compass model are hinted in Figure 7, where the squared slowness gradients of WRI* and FWI (computed with respect to the background model in Figure 6b) are compared. These are obtained by filtering the data according to a narrow frequency band centered at $5 \mathrm{~Hz}$ (a similar result can be obtained by working directly in the frequency domain, Peters et al., 2014). We observe that FWI produces an erroneous update in the water layer, while WRI* points in the correct direction.

For the inversion, we adopt a multiscale strategy (Bunks et al., 1995), by filtering and inverting data components of increasing frequency content, from 5 $\mathrm{Hz}$ to $20 \mathrm{~Hz}$. This is achieved by preconditioning both data and synthetics. For simplicity, we do not adapt the model grid to the varying frequency. At each of these sequential stages, we perform l-BFGS optimization for 20 iterations and use the result as the starting guess for the next step. The process is repeated with multiple sweeps across the frequency range, by repeating the multiscale inversion twice and restarting from the lowest frequency when the first pass is completed. Multiple sweeps are here necessary due to the high-low velocity inversion specific of the BG Compass model. During the first pass, the inversion develops a spurious reflector across the high-velocity layer located between 500 $\mathrm{m}$ and $1000 \mathrm{~m}$ deep, and subsequent passes are designed to remove it. The final results for WRI* and FWI are collected in Figure 8. For reference, see also Peters et al. (2014) for frequency-domain WRI inversion results on a similar model.

Notably, the poor update generated by FWI within the water layer eventually leads to the local minimum in Figure 8a (for FWI, we report the result after only one data sweep). The WRI* result is shown in Figure 8b. Clearly, WRI* is able to correct for the water layer and resolve the high-velocity/low-velocity sequence at $1 \mathrm{Km}$ depth. The deeper part of the model can be improved, in principle, by further optimization or pseudo-Hessian preconditioning via incident field energy (see van Leeuwen and Herrmann, 2013, for a simple Gauss-Newton scheme).

As a further quality control assessment, in Figure 9 we compare RTM results obtained from the initial velocity model and from the the WRI* inversion. We 


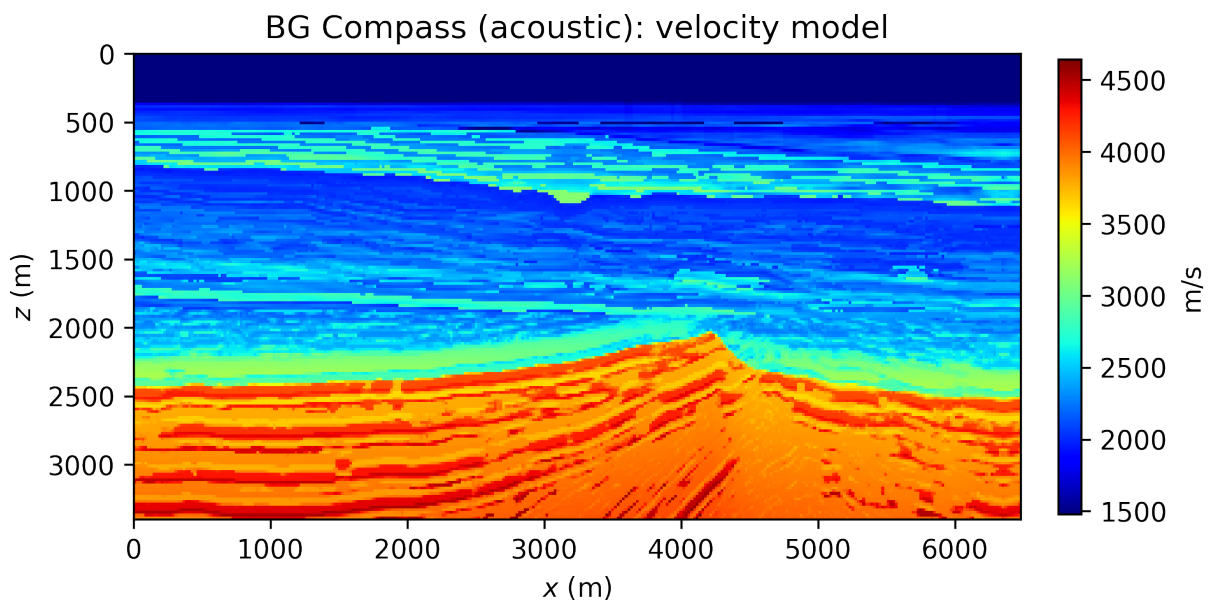

(a)

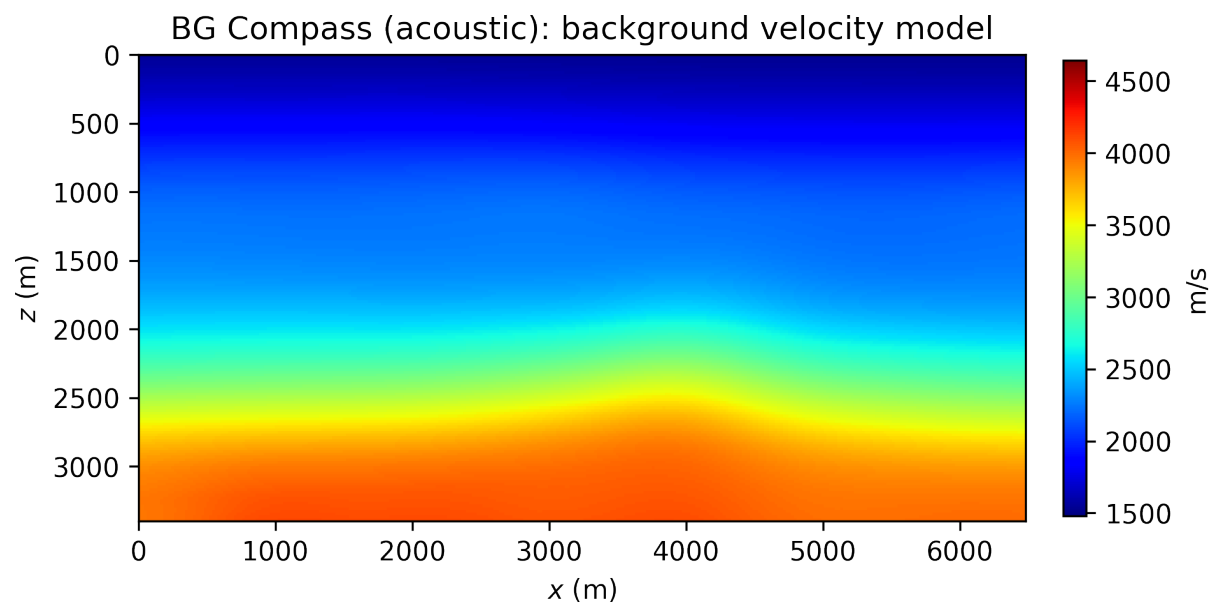

(b)

Figure 6: BG Compass, acoustic model: (a) velocity model, (b) background model.

migrate synthetic data generated with a Ricker wavelet of $20 \mathrm{~Hz}$ peak frequency (while we used $10 \mathrm{~Hz}$ peak frequency data for inversion), the rest of the sourcereceiver acquisition setting remains the same. The RTM image obtained with the WRI* inverted model is resolved with higher resolution at shallow depth, while the deeper reflectors more accurately migrated.

Inversion with inaccurate modeling assumptions Here, we consider a TTI anisotropic version of the acoustic BG Compass model, previously shown in Figure 6a. The model is rescaled to simplify the problem by mitigating the effect 


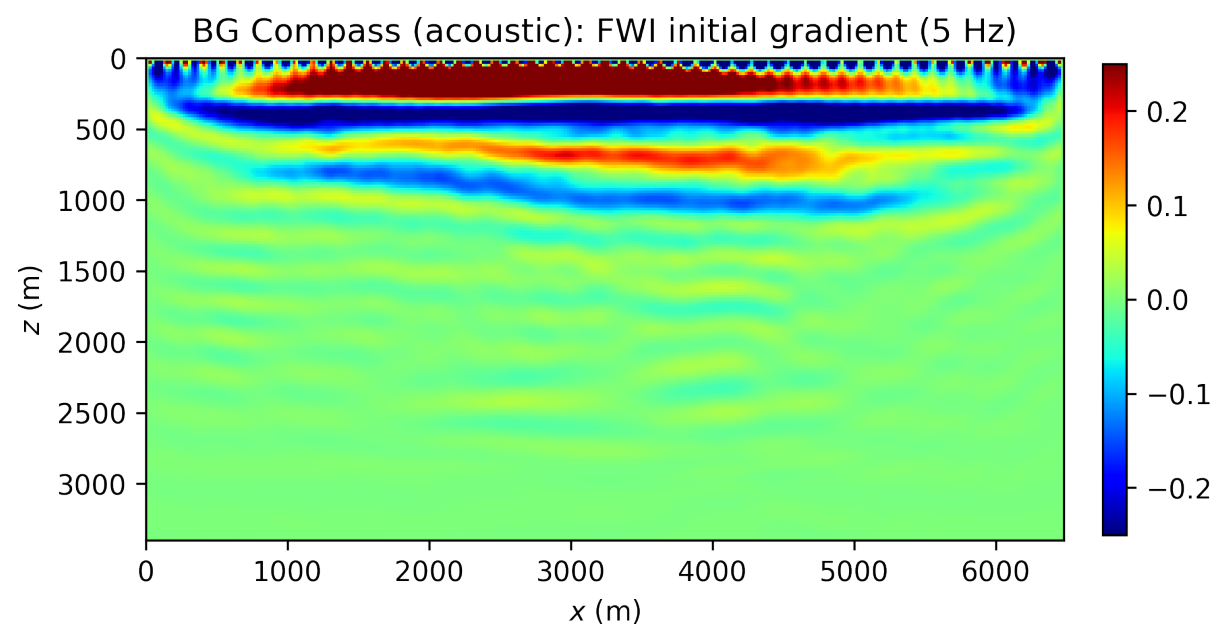

(a)

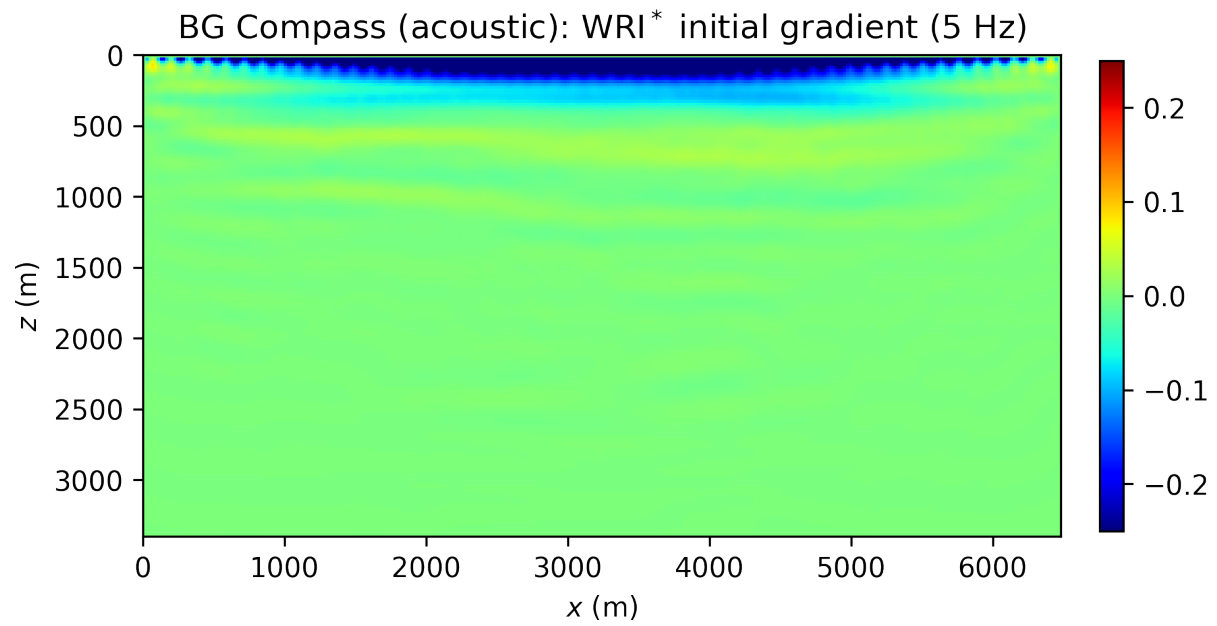

(b)

Figure 7: Gradient comparison for a single frequency component $5 \mathrm{~Hz}$ (with respect to homogeneous squared slowness parameters): (a) FWI (normalized), (b) WRI* (normalized). The FWI gradient seeks to update the water layer in the wrong direction.

of the velocity kickback. Artifacts will now appear deeper in the model. The Thomsen parameters $\varepsilon$ and $\delta$ are synthesized from the velocity model, and dip angles inferred from the orientation of the layers. The TTI model is presented in Figure 10.

Data are generated according to the same settings described for the acoustic BG Compass model (note, however, that the TTI model size is different). 


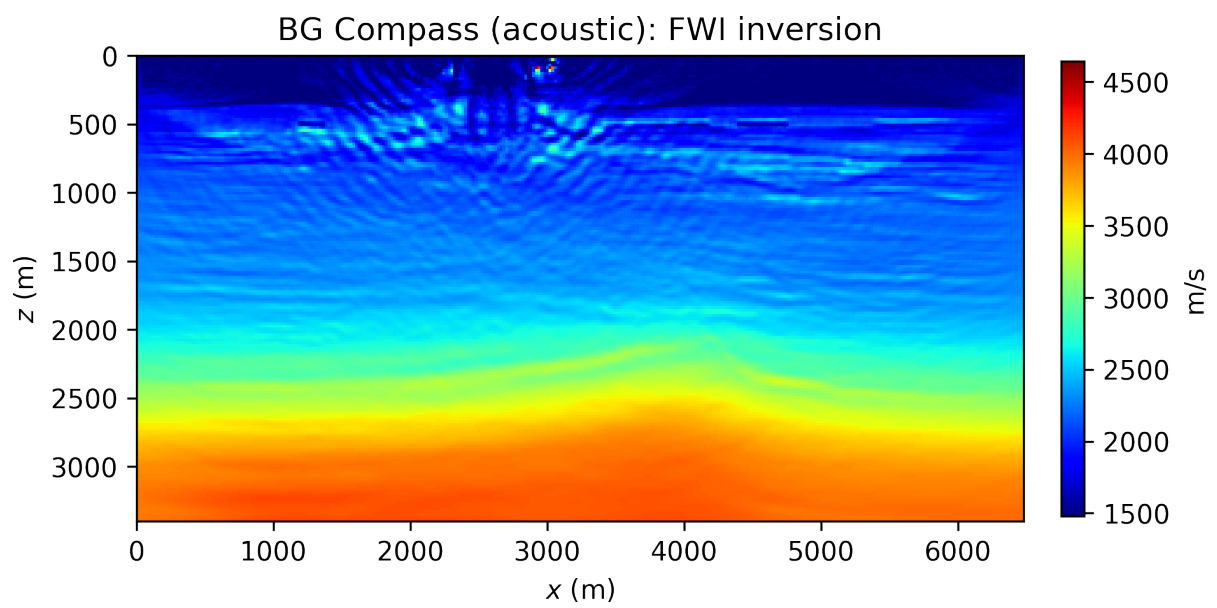

(a)

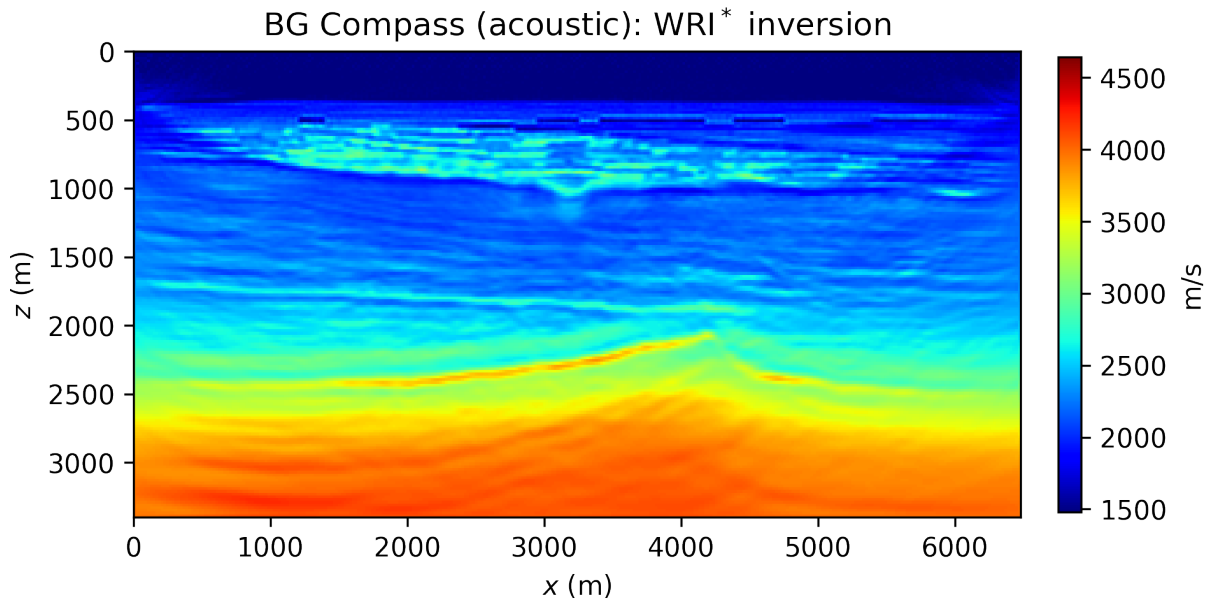

(b)

Figure 8: Acoustic BG Compass inversion result for: (a) FWI, (b) WRI*.

The inversion is performed with data generated with a Ricker wavelet of peak frequency $10 \mathrm{~Hz}$ (with low frequencies filtered out), and the optimization is carried out by 20 iterations of the spectral projected gradient method (SPG) of Birgin et al. (2000) (the projection here bounding velocity values). We compare WRI* and FWI results by initializing the inversion with smooth models obtained from the true velocity map. The level of smoothness is gradually increased to assess the robustness of the two methods. The anisotropic parameters are smoothed and kept fixed for all the experiments and throughout the inversion. The inconsistency between true and assumed TTI parameters will be the source of modeling inaccuracy with which we test the resilience of the velocity model 


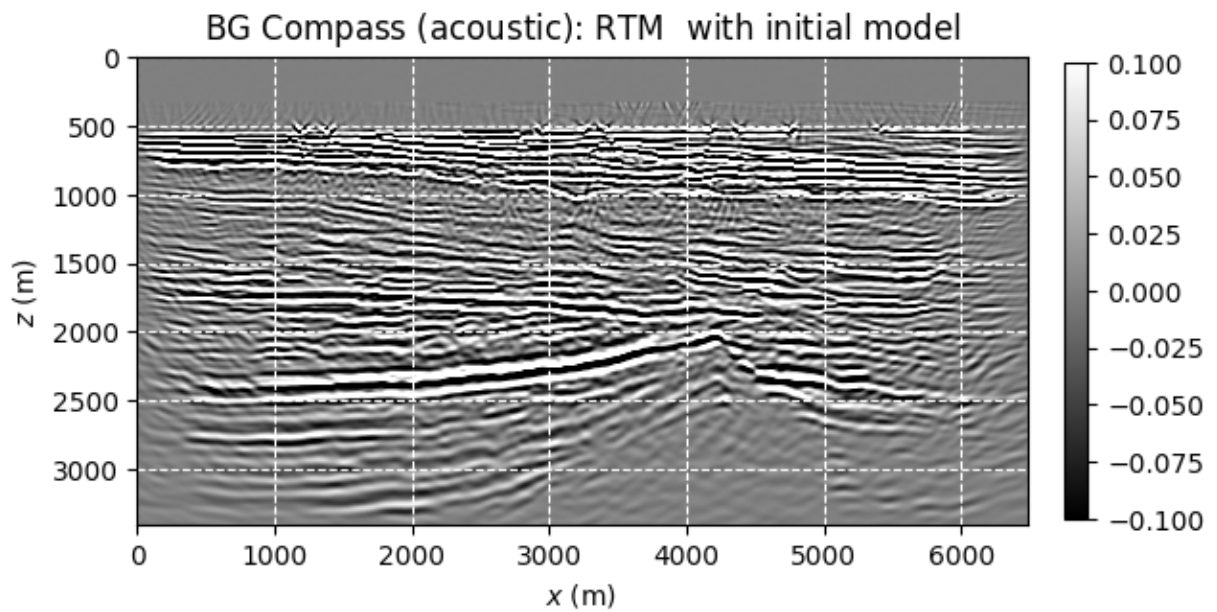

(a)

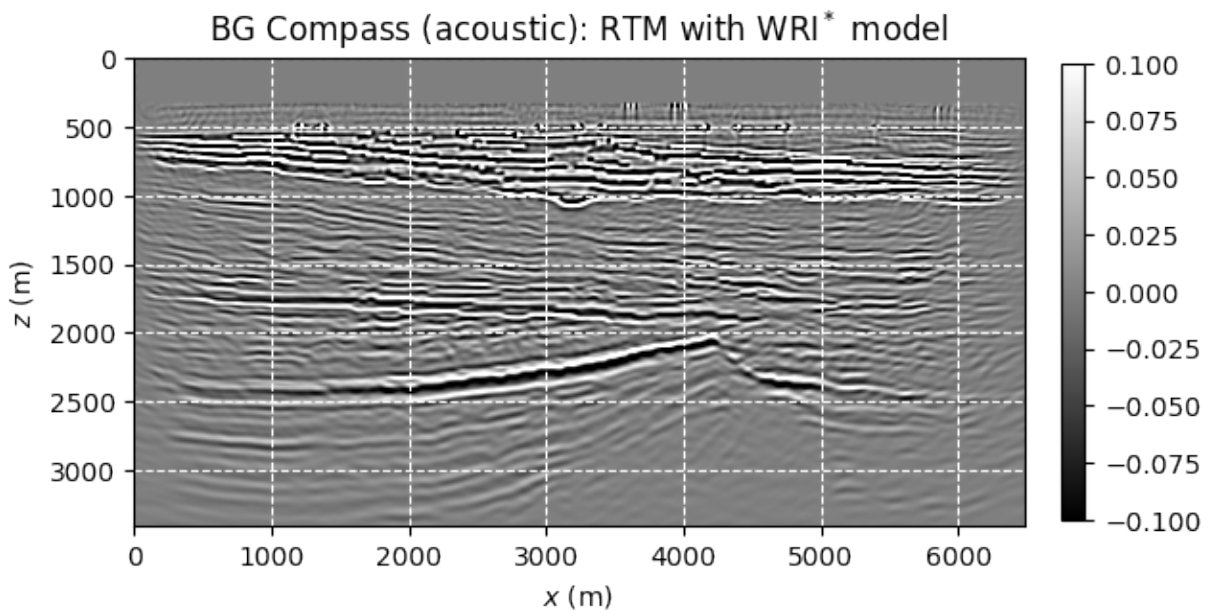

(b)

Figure 9: Reverse time migration results (normalized for display purposes) for the BG Compass model, corresponding to different background velocity models: (a) starting model (see Figure 6b), (b) inversion result for WRI* (see Figure 8b). A grid is overlayed in white to aid the comparison.

recovery with WRI* and FWI. Smoothing is carried out via Gaussian filtering, modulated by the dimentionless standard deviation $\sigma$ obtained via normalization with the grid spacing. An example of smoothed TTI model is depicted in Figure 11.

The inversion results for WRI* and FWI are displayed in Figure 12. We notice that the starting model and wrong assumptions about anisotropy have an effect on $\mathrm{WRI}^{*}$ results, especially in resolving the shallow high-velocity layer and the 


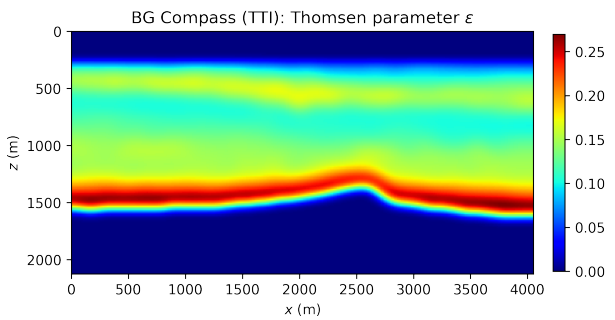

(a)

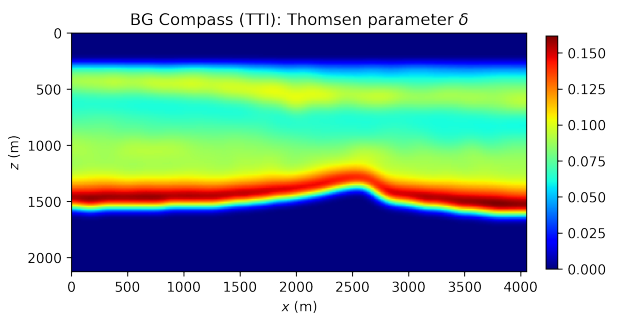

(b)

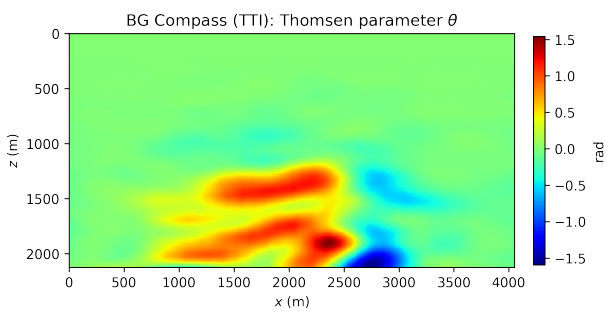

(c)

Figure 10: BG Compass, TTI model: (a) Thomsen parameter $\varepsilon$, (b) Thomsen parameter $\delta$, (c) anisotropy dip angle $\theta$. Refer to Figure 6a for the velocity model.

deeper part of the model. Despite this, the WRI* results remain relatively stable with respect to the starting guess, the major difference being a loss of resolution with depth with worse starting models. More notably, they are consistently better than the FWI results, which are instead heavily affected by this choice.

\subsection{Preliminary 3D results}

This numerical experiment aims at the gradient comparison between WRI* and FWI for a small acoustic 3D problem modeled after the previous low-velocity lens example in $2 \mathrm{D}$, now defined on a $51 \times 51 \times 51$ grid. We consider transmission data generated by $8 \times 8$ evenly sampled sources located at $z=120 \mathrm{~m}$, and $91 \times 91$ receivers at $z=1890 \mathrm{~m}$. We employ a Ricker source wavelet of 5 $\mathrm{Hz}$ peak frequency, but the frequency components below $3 \mathrm{~Hz}$ are filtered out. 


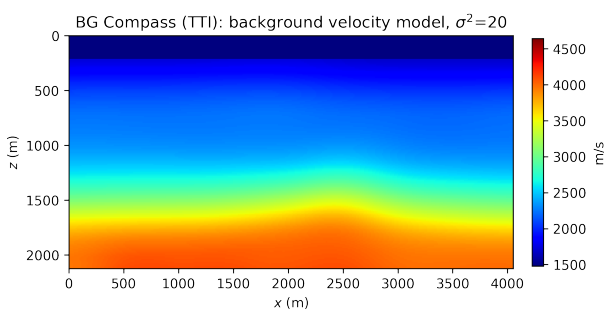

(a)

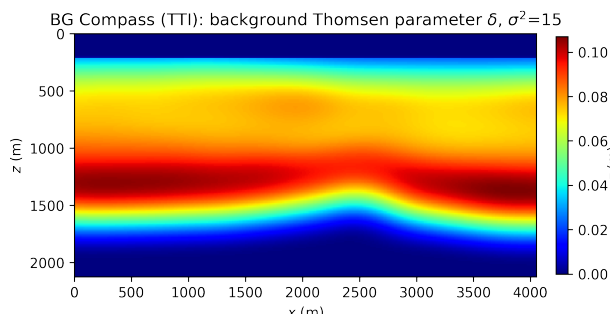

(c)

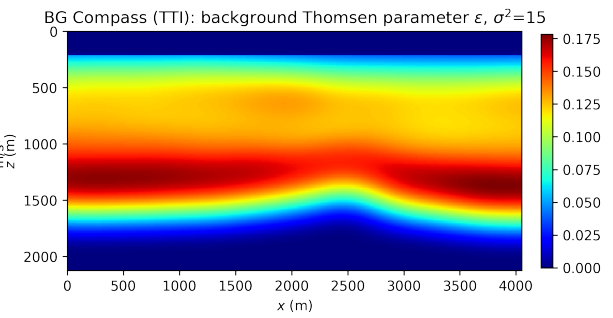

(b)

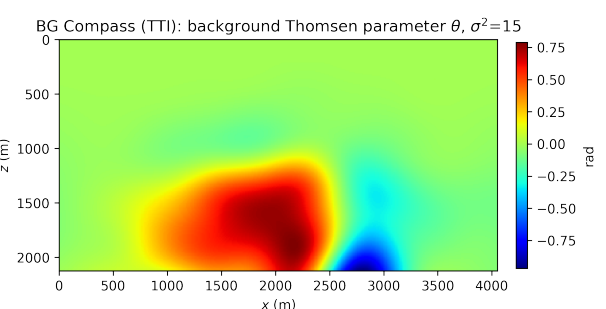

(d)

Figure 11: BG Compass, TTI starting model: (a) velocity model, (b) Thomsen parameter $\varepsilon$, (c) Thomsen parameter $\delta$, (b) anisotropy dip angle $\theta$. In the inversion, the anisotropic parameters $\varepsilon, \delta$, and $\theta$ here displayed are kept fixed, and only the velocity is updated. Note that the anisotropic parameters are smoothed version of the true model in Figure 10. Smoothing is performed via Gaussian filtering with the indicated standard deviation $\sigma$ (normalized with respect to the grid spacing).

In Figure 13a, we depict the difference between the true model and an initial homogeneous model.

As in the 2D case, the gradient comparison for WRI* and FWI in Figure 13 shows that WRI*, unlike FWI, leads to a correct velocity model update.

\section{Discussion}

The application of modern seismic inversion tools to field data has been curbed by the need for tremendous computational resources, which are required for wave simulations on extremely large 3D grids. Moreover, classical acoustic models are being discarded in favor of pseudo-acoustic (now standard in industrial imaging) or elastic simulators to account for anisotropic phenomena in field data. Less simplistic modeling inevitably adds to the computational complexity of the problem. Be that as it may, the oil and gas industry manages to employ FWI on a routine basis, thanks to efficient time-domain simulators, and its business value is widely acknowledged. Unfortunately, the success of FWI is highly dependent on a lengthy preprocessing phase aimed at the retrieval of a 


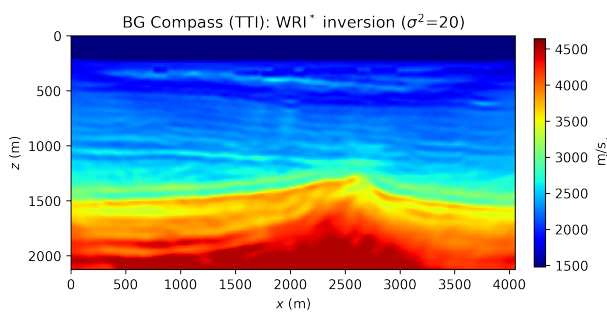

(a)

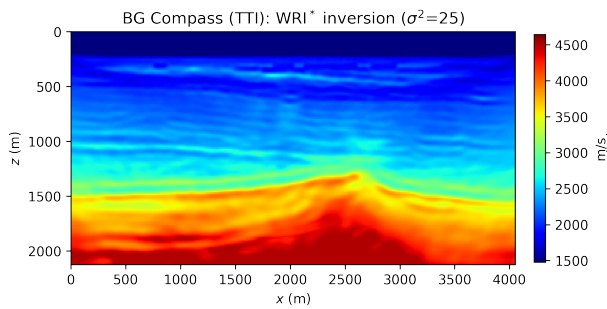

(c)

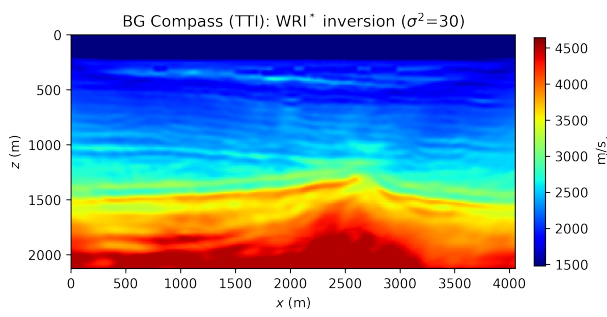

(e)

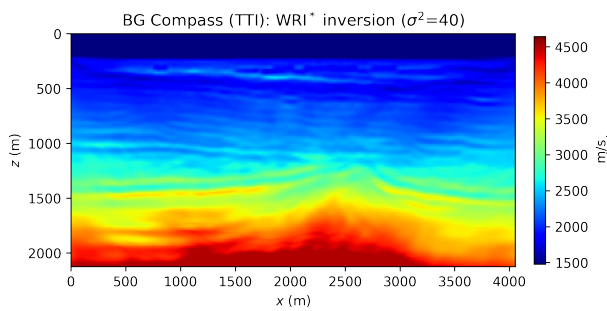

(g)

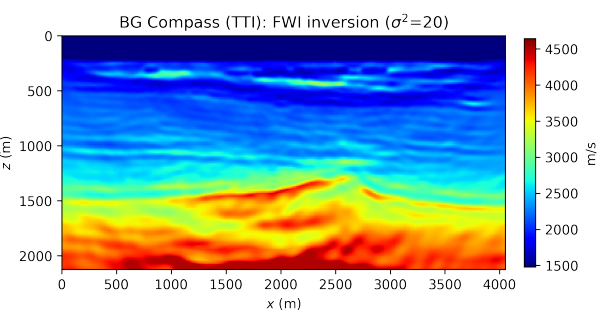

(b)

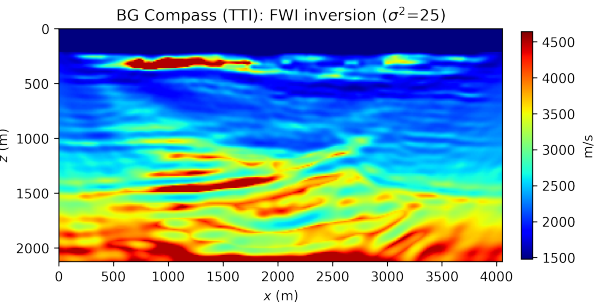

(d)

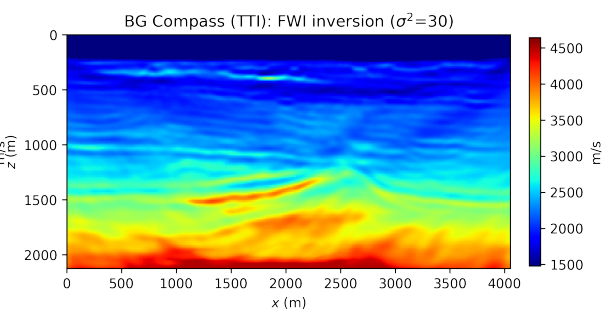

(f)

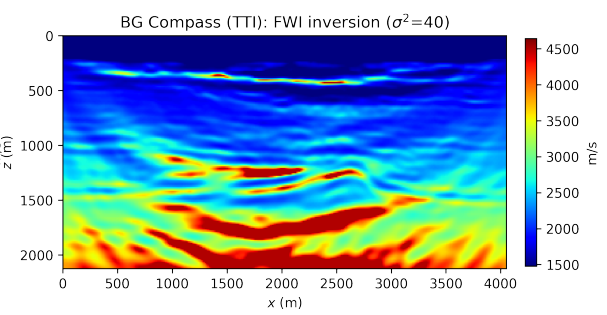

(h)

Figure 12: BG Compass, TTI inversion results acquired with starting velocity models obtained from smoothing the ground truth with Gaussian filters with varying standard deviations $\sigma$ normalized by grid spacing (the anisotropy parameters are kept fixed as in Figures 11b, 11c, 11d): (a,b) WRI* and FWI results (respectively) for $\sigma^{2}=20,(\mathrm{c}, \mathrm{d}) \sigma^{2}=25,(\mathrm{e}, \mathrm{f}) \sigma^{2}=30,(\mathrm{~g}, \mathrm{~h}) \sigma^{2}=40$. The physical model underlying the inversion always assume inaccurate anisotropy, but WRI* delivers consistently better result than FWI and remain relatively stable with respect to the starting guess. FWI, on the other hand, does increasingly get worse as the starting model is further from the ground truth. 


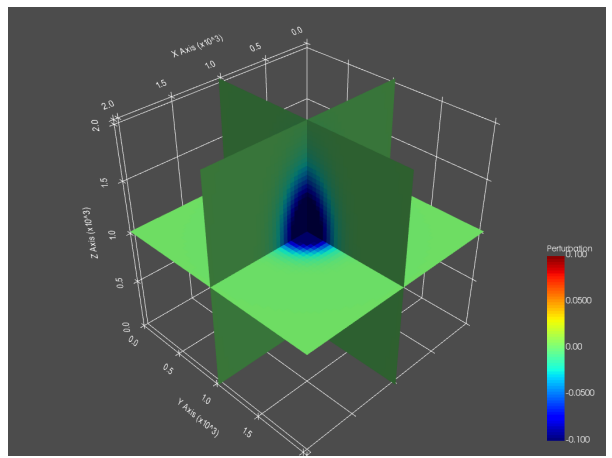

(a)

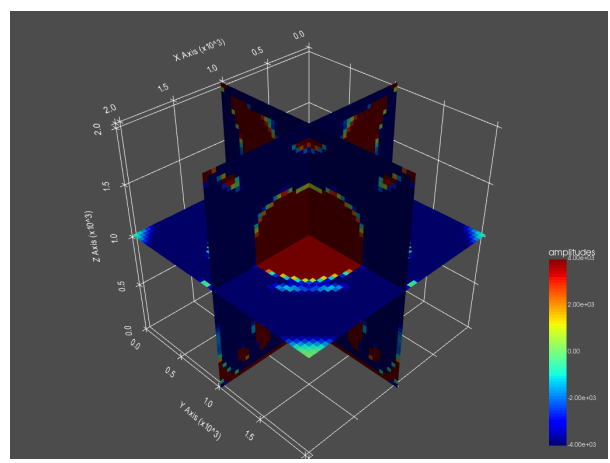

(b)

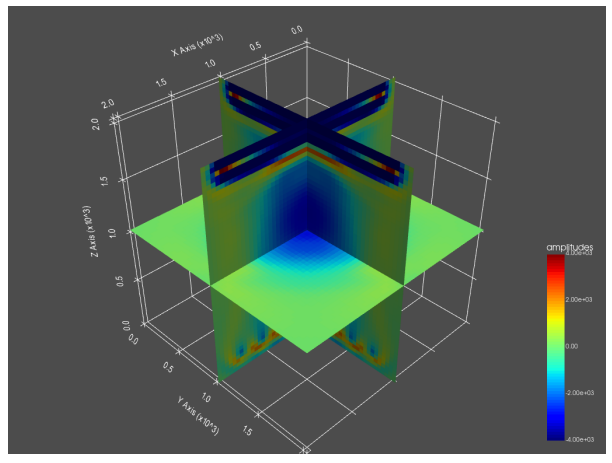

(c)

Figure 13: 3D low-velocity lens model: (a) true model perturbation (with respect to an homogeneous background), (b) initial gradient for FWI, (c) initial gradient for WRI*. As in the 2D case, the FWI gradient points to the wrong direction, while the $\mathrm{WRI}^{*}$ gradient allows for the correct update. 
kinematically accurate background and starting guess for seismic inversion.

The main objective of this work is to dispense with the need for accurate initial velocity models in seismic inversion. Despite the fact that many solutions exist focusing on robustness with respect to local minima, they do not scale efficiently with grid size and are not relevant for real-world applications. One such example is WRI. WRI is, nonetheless, an attractive method that is not only robust with respect to the starting guess, but also has the potential to handle moderate inaccuracies in some of the physical parameters that are typically kept fixed during inversion. Indeed, WRI hinges on a relaxed wave equation, whose solution, however, can only be approximated iteratively in 3D with considerable computational costs.

We presented a novel method, WRI* which is both scalable and robust. It is based on a denoising reformulation of WRI, which amounts to the minimization of the wave equation error subject to the data misfit being less than a known noise level. The resulting saddle-point problem involves the usual model unknowns, such as squared slowness, and Lagrangian multipliers having the same dimension as data. We stress that all the computations needed for the evaluation of the Lagrangian and its gradients require standard modeling in time domain, which can be attained for realistically sized problems (contrary to frequency domain formulations). Since the dual variables can be stored in memory, we might in principle devise a joint optimization scheme. In this paper, however, we approximated the Lagrangian multipliers by the scaled data residual in order to reduce complexity (a more accurate assessment of the trade-off between multiplier approximation and reconstruction quality is left for future studies).

We demonstrated, through extensive numerical experimentation, that the resulting method is consistently more robust than FWI against local minima. We also tested the tolerance of the method toward faulty modeling assumptions, e.g. when seismic data obtained with anisotropic modeling is being matched with predictions based on the wrong anisotropic model. WRI*, which employs a relaxation of the wave equation, produces better results than FWI, under the same conditions.

In general, the results obtained from WRI* do depend on the particular choice of hyperparameters such as the data noise level $\epsilon$, defined in equation (19). This parameter governs the trade-off between wave equation error and data misfit and is akin to the role of the weighting parameter $\lambda$, in equation (5), for conventional WRI. We find that decreasing its value tends to produce lower resolution results. On the other hand, choosing a small value for $\epsilon$ (or even $\epsilon=0$ ) results in relaxed physics and is beneficial for local minimum avoidance. In our experience, WRI* retains the same ability of WRI to circumvent local minima, but might produce less qualitative results, especially in terms of resolution. We note that conventional WRI is not as affected as $\mathrm{WRI}^{*}$ by the choice of $\lambda$. The fundamental reason for this phenomenon is rooted in the inexact, but relatively inexpensive, dual variable approximation in equation (27), as opposed to equation (25). Taking into account the dependency of the approximated dual variable with respect to the model parameter, as in equation (29), is designed to partially compensate for this behavior (Ablin et al., 2020). We then advocate 
for either a continuation strategy for $\epsilon$, by starting with $\epsilon=0$ and increasing its value during the inversion (as hinted by the Marmousi experiment in this paper), proper preconditioning (as in the Gauss-Newton approach in van Leeuwen and Herrmann, 2013), or a sequential approach consisting of a WRI* phase followed by FWI (albeit, at the cost of potentially losing the robustness of WRI* towards modeling hinging on inaccurate physical parameters). Another approach might consist of iteratively solving for the optimal dual variable, but we envisage this can be only feasible in combination with stochastic optimization, where simultaneous sources are considered.

From a computational perspective, WRI* requires roughly twice as much the cost needed for FWI, per objective evaluation or gradient calculation. Given the gain in robustness previously discussed, we deem this extra cost acceptable in the many situations where FWI would normally fail, since the result improvement easily offsets the extra computations. Naturally, hybrid combinations of WRI* and FWI are also possible, as shown here, to amortize these costs.

\section{Conclusion}

We proposed a seismic inversion method whose main strength is being both robust with respect to local minima and scalable to $3 \mathrm{D}$. Traditional methods are either scalable but prone to cycle skipping, like full waveform inversion, or less sensitive to the starting model but unfeasible for large problems, like wavefield reconstruction inversion. Our proposal, despite being a reformulation of wavefield reconstruction inversion, can leverage on modern time-domain solvers, which is key to tackle realistically sized problems. Numerical experiments indicate that our proposal is consistently superior to full waveform inversion when cycle skipping occurs. Moreover, it benefits from a relaxed formulation of the wave equation, and is more resilient than full waveform inversion with respect to starting models when inaccurate modeling assumptions are made. A comparison with conventional wavefield reconstruction inversion highlights competitive results, albeit with some loss of resolution, depending on the choice of hyperparameters like the estimated data noise level. It has been shown that these shortcomings can be easily avoided with an hybrid scheme involving full waveform inversion and/or pseudo-Hessian preconditioning. Our method is mature for $3 \mathrm{D}$, but the computational effort required is twice as much what is prescribed by full waveform inversion (per gradient calculation). This extra cost can be reduced by aforementioned hybrid schemes and stochastic optimization.

\section{Related material}

A Julia implementation of the method herein described can be found in the following repository: https://github.com/slimgroup/JUDI.jl/tree/master/examples/twri 


\section{Acknowledgments}

This work has been implemented in Julia and leverages the Devito framework for seismic modeling, which allows automatic generation of highly-optimized finitedifference $\mathrm{C}$ code, given only a symbolic representation of the wave equation (Louboutin et al., 2019; Luporini et al., 2018). From Julia, we interface to Devito through the JUDI package (Witte et al., 2019a).

\section{References}

Ablin, P., G. Peyré, and T. Moreau, 2020, Super-efficiency of automatic differentiation for functions defined as a minimum: Technical report.

Aghamiry, H. S., A. Gholami, and S. Operto, 2019, Improving full-waveform inversion by wavefield reconstruction with the alternating direction method of multipliers: Geophysics, 84, R139-R162.

Akcelik, V., G. Biros, and O. Ghattas, 2002, Parallel multiscale Gauss-NewtonKrylov methods for inverse wave propagation: SC'02: Proceedings of the 2002 ACM/IEEE Conference on Supercomputing, IEEE, 41-41.

Alkhalifah, T., 2000, An acoustic wave equation for anisotropic media: GEOPHYSICS, 65, 1239-1250.

Anagaw, A. Y., and M. D. Sacchi, 2012, Edge-preserving seismic imaging using the total variation method: Journal of Geophysics and Engineering, 9, 138-146.

Baysal, E., D. D. Kosloff, and J. W. C. Sherwood, 1983, Reverse time migration: Geophysics, 48, 1514-1524.

Beydoun, W. B., and A. Tarantola, 1988, First Born and Rytov approximations: Modeling and inversion conditions in a canonical example: Journal of the Acoustical Society of America, 83, 1045-1055.

Biondi, B., and A. Almomin, 2014, Simultaneous inversion of full data bandwidth by tomographic full-waveform inversion: Geophysics, 79, WA129-WA140.

Birgin, E. G., J. M. Martínez, and M. Raydan, 2000, Nonmonotone Spectral Projected Gradient Methods on Convex Sets: SIAM Journal on Optimization, 10, 1196-1211.

Bjorck, A., 1996, Numerical Methods for Least Squares Problems: Society for Industrial and Applied Mathematics.

Bozdag, E., J. Trampert, and J. Tromp, 2011, Misfit functions for full waveform inversion based on instantaneous phase and envelope measurements: Geophys. J. Int., 185, 845-870.

Bunks, C., F. M. Saleck, S. Zaleski, and G. Chavent, 1995, Multiscale seismic waveform inversion: GEOPHYSICS, 60, 1457-1473.

Chambolle, A., and T. Pock, 2011, A first-order primal-dual algorithm for convex problems with applications to imaging: Journal of mathematical imaging and vision, 40, 120-145.

da Silva, N. V., and G. Yao, 2017, Wavefield reconstruction inversion with a multiplicative cost function: Inverse problems, 34, 015004.

Duveneck, E., P. Milcik, P. M. Bakker, and C. Perkins, 2008, in Acoustic VTI 
wave equations and their application for anisotropic reverse-time migration: $2186-2190$.

Esser, E., L. Guasch, T. van Leeuwen, A. Y. Aravkin, and F. J. Herrmann, 2018, Total-variation regularization strategies in full-waveform inversion: SIAM Journal on Imaging Sciences, 11, 376-406.

Golub, G., and V. Pereyra, 2003, Separable nonlinear least squares: the variable projection method and its applications: Inverse problems, 19, R1.

Grechka, V., L. Zhang, and J. W. I. Rector, 2004, Shear waves in acoustic anisotropic media: GEOPHYSICS, 69, 576-582.

Griewank, A., and A. Walther, 2008, Evaluating derivatives: principles and techniques of algorithmic differentiation: Siam, 105.

Guasch, L., M. Warner, and C. Ravaut, 2019, Adaptive waveform inversion: Practice: GEOPHYSICS, 84, R447-R461.

Haber, E., U. M. Ascher, and D. Oldenburg, 2000, On optimization techniques for solving nonlinear inverse problems: Inverse problems, 16, 1263.

Herrmann, F. J., T. van Leeuwen, A. Y. Aravkin, and H. Calandra, 2013, In which domain should we measure the misfit for robust full waveform inversion?: 75th EAGE Conference \& Exhibition incorporating SPE EUROPEC 2013, European Association of Geoscientists \& Engineers, cp-348.

Huang, G., R. Nammour, and W. W. Symes, 2017, Full-waveform inversion via source-receiver extension: Geophysics, 82, R153-R171.

$\ldots$, 2018, Volume source-based extended waveform inversion: Geophysics, 83, R369-R387.

Knibbe, H., C. Vuik, and C. W. Oosterlee, 2016, Reduction of computing time for least-squares migration based on the Helmholtz equation by graphics processing units: Computational Geosciences, 20, 297-315.

Louboutin, M., M. Lange, F. Luporini, N. Kukreja, P. A. Witte, F. J. Herrmann, P. Velesko, and G. J. Gorman, 2019, Devito (v3.1.0): an embedded domainspecific language for finite differences and geophysical exploration: Geoscientific Model Development, 12, 1165-1187.

Luo, Y., and G. T. Schuster, 1991, Wave-equation traveltime inversion: GEOPHYSICS, 56, 645-653.

Luporini, F., M. Lange, M. Louboutin, N. Kukreja, J. Hückelheim, C. Yount, P. Witte, P. H. J. Kelly, F. J. Herrmann, and G. J. Gorman, 2018, Architecture and performance of Devito, a system for automated stencil computation: CoRR, abs/1807.03032.

Luporini, F., M. Lange, M. Louboutin, N. Kukreja, J. Hückelheim, C. Yount, P. A. Witte, P. H. J. Kelly, G. J. Gorman, and F. J. Herrmann, 2020, Architecture and performance of devito, a system for automated stencil computation: ACM Trans. Math. Softw., 46. ((ACM Trans. Math. Softw.)).

Métivier, L., R. Brossier, Q. Mérigot, E. Oudet, and J. Virieux, 2016, Measuring the misfit between seismograms using an optimal transport distance: application to full waveform inversion: Geophys. J. Int., 205, 345-377.

Nocedal, J., and S. Wright, 2006, Numerical optimization: Springer Science \& Business Media.

Peters, B., and F. J. Herrmann, 2017, Constraints versus penalties for edge- 
preserving full-waveform inversion: The Leading Edge, 36, 94-100. ((The Leading Edge)).

— , 2019, A numerical solver for least-squares sub-problems in 3D wavefield reconstruction inversion and related problem formulations: SEG Technical Program Expanded Abstracts, 1536-1540. ((SEG, San Antonio)).

Peters, B., F. J. Herrmann, and T. van Leeuwen, 2014, Wave-equation Based Inversion with the Penalty Method - Adjoint-state Versus Wavefield- reconstruction Inversion: 76th EAGE Conference and ..., 16-19.

Peters, B., B. R. Smithyman, and F. J. Herrmann, 2018, Projection methods and applications for seismic nonlinear inverse problems with multiple constraints: Geophysics, 84, R251-R269.

Rockafellar, R. T., 1970, Convex analysis: Princeton university press.

Sharan, S., R. Wang, and F. J. Herrmann, 2018, Fast sparsity-promoting microseismic source estimation: Geophysical Journal International, 216, 164-181.

Shin, C., and Y. H. Cha, 2008, Waveform inversion in the Laplace domain: Geophys. J. Int., 173, 922-931.

— 2009, Waveform inversion in the Laplace-Fourier domain: Geophys. J. Int., 177, 1067-1079.

Shipp, R. M., and S. C. Singh, 2002, Two-dimensional full wavefield inversion of wide-aperture marine seismic streamer data: Geophys. J. Int., 151, 325-344.

Sirgue, L., J. T. Etgen, and U. Albertin, 2008, 3D frequency domain waveform inversion using time domain finite difference methods: 70th EAGE Conference and Exhibition incorporating SPE EUROPEC 2008, European Association of Geoscientists \& Engineers, cp-40.

Sirgue, L., and R. G. Pratt, 2004, Efficient waveform inversion and imaging: A strategy for selecting temporal frequencies: Geophysics, 69, 231-248.

Sun, B., and T. Alkhalifah, 2019, The application of an optimal transport to a preconditioned data matching function for robust waveform inversion: Geophysics, 84, R923-R945.

Symes, W. W., 2007, Reverse time migration with optimal checkpointing: Geophysics, 72, SM213-SM221.

- 2008, Migration velocity analysis and waveform inversion: Geophysical Prospecting, 56, 765-790.

— 2020a, Full Waveform Inversion by Source Extension: Why it works.

- 2020b, Wavefield Reconstruction Inversion: an example: Inverse Problems.

Tarantola, A., and B. Valette, 1982, Generalized nonlinear inverse problems solved using the least squares criterion: Reviews of Geophysics, 20, 219-232. van den Berg, P. M., and R. E. Kleinman, 1997, A contrast source inversion method: Inverse problems, 13, 1607.

van Leeuwen, T., and F. J. Herrmann, 2013, Mitigating local minima in fullwaveform inversion by expanding the search space: Geophysical Journal International, 195, 661-667.

—_ 2014, 3D frequency-domain seismic inversion with controlled sloppiness: SIAM Journal on Scientific Computing, 36, S192-S217.

van Leeuwen, T., and W. A. Mulder, 2010, A correlation-based misfit criterion for wave-equation traveltime tomography: Geophysical Journal International, 
182, $1383-1394$.

Virieux, J., and S. Operto, 2009, An overview of full-waveform inversion in exploration geophysics: Geophysics, 74, WCC1-WCC26.

Wang, C., D. Yingst, P. Farmer, and J. Leveille, 2016, Full-waveform inversion with the reconstructed wavefield method, in SEG Technical Program Expanded Abstracts 2016: Society of Exploration Geophysicists, 1237-1241.

Wang, R., and F. J. Herrmann, 2017a, A denoising formulation of full-waveform inversion: SEG Technical Program Expanded Abstracts, 1594-1598.

— 2017b, A denoising formulation of Full-Waveform Inversion, in SEG Technical Program Expanded Abstracts 2017: Society of Exploration Geophysicists, 1594-1598.

Wang, S., M. V. de Hoop, and J. Xia, 2011, On 3D modeling of seismic wave propagation via a structured parallel multifrontal direct Helmholtz solver: Geophysical Prospecting, 59, 857-873.

Warner, M., and L. Guasch, 2016, Adaptive waveform inversion: Theory: GEOPHYSICS, 81, R429-R445.

Witte, P. A., M. Louboutin, N. Kukreja, F. Luporini, M. Lange, G. J. Gorman, and F. J. Herrmann, 2019a, A large-scale framework for symbolic implementations of seismic inversion algorithms in Julia: GEOPHYSICS, 84, F57-F71.

Witte, P. A., M. Louboutin, F. Luporini, G. J. Gorman, and F. J. Herrmann, 2019b, Compressive least-squares migration with on-the-fly fourier transforms: Geophysics, 84, R655-R672. ((Geophysics)).

Witte, P. A., M. Louboutin, H. Modzelewski, C. Jones, J. Selvage, and F. J. Herrmann, 2020, An event-driven approach to serverless seismic imaging in the cloud: IEEE Transactions on Parallel and Distributed Systems, 31, 2032-2049. ((IEEE Transactions on Parallel and Distributed Systems)).

Yang, Y., B. Engquist, J. Sun, and B. F. Hamfeldt, 2018, Application of optimal transport and the quadratic wasserstein metric to full-waveform inversion: GEOPHYSICS, 83, R43-R62. 